\title{
Second-order corrected likelihood for nonlinear panel models with fixed effects
}

\author{
Geert Dhaene* $\quad$ Yutao Sun $^{\dagger}$
}

October 11, 2019

\begin{abstract}
We propose a second-order correction for nonlinear fixed-effect panel models. The correction is made via the log-likelihood function. It removes the two leading terms of the bias of the log-likelihood that arises from estimating the fixed effects. Maximizing the corrected likelihood gives a second-order bias-corrected estimator, with bias $O\left(T^{-3}\right)$, where $T$ is the number of time periods. The corrected likelihood also gives second-order corrected test statistics. The correction applies to general nonlinear fixed-effect models with independent observations. The bias correction properties are confirmed in simulations for binary-choice models.
\end{abstract}

JEL Classification: C23

Keywords: Nonlinear panel data models; fixed effects; incidental parameter problem; bias correction

\section{Introduction}

Panel data are becoming ever more important in economic studies. In panel studies, researchers often attempt to capture unobserved individual heterogeneity by introducing individual-specific parameters, or "fixed effects", in the model. When the number of time periods in the data set is small, however, nonlinear models with fixed-effect parameters may give maximum likelihood (ML) estimates that are severely biased. This is known as the incidental parameter problem (IPP) of Neyman and Scott (1948). Lancaster (2000) surveys the IPP and various approaches trying to solve it. To outline the problem, let the data set have $N$ cross-sectional units, indexed by $i=1, \cdots, N$, and $T$ time periods, indexed by $t=1, \cdots, T$. Suppose, as is common in micro-panels, that $N$ is large and $T$ is small. This situation is well approximated by asymptotics with $N \rightarrow \infty$ and $T$ fixed. Let $\log f\left(Y_{i t} ; \theta, \alpha_{i}\right)$ be the $\log$-likelihood associated with observation $Y_{i t}$ (possibly conditional on observed covariates $X_{i t}$ ). Here $\theta$ is the vector of parameters that apply to all observations $i, t$ (so $\theta$ is the "common" parameter) and $\alpha_{1}, \cdots, \alpha_{N}$ are fixed-effect (nuisance) parameters, or "incidental" parameters as Neyman and Scott (1948) called them. Now $\widehat{\alpha}_{i}$, the ML estimator of $\alpha_{i}$, only uses the data from the $i$ th individual. Therefore, when $T$ is fixed, $\widehat{\alpha}_{i}$ remains random for every $i$ even

\footnotetext{
*Faculty of Economics and Business, KU Leuven, Leuven (3000), Belgium. Email: geert.dhaene@kuleuven.be.

${ }^{\dagger}$ Corresponding author. School of Economics, Northeast Normal University, Changchun (130117), China, and Erasmus School of Economics, Erasmus University Rotterdam, Rotterdam (3000 DR), the Netherlands. Email: sun@ese.eur.nl.
} 
as $N \rightarrow \infty$. In many models, this introduces a bias into the log-likelihood function in the sense that the ML estimator $\widehat{\theta}$ converges to a point $\theta_{T}$ that differs from $\theta_{0}$, the true value of $\theta$. One may view $\theta_{0}$ as the maximizer of the probability limit, as $N \rightarrow \infty$, of the log-likelihood that depends on the true values of the fixed effects; and $\theta_{T}$ as the maximizer of the probability limit, as $N \rightarrow \infty$, of the log-likelihood that depends on the estimates of the fixed effects. The two probability limits are generically not the same and, therefore, their maximizers, $\theta_{0}$ and $\theta_{T}$, are also different. The bias of $\theta$ is $O\left(T^{-1}\right)$. When $T \rightarrow \infty$, every $\widehat{\alpha}_{i}$ converges to the corresponding true value $\alpha_{i 0}$, and so does $\widehat{\theta}$. However, when $N, T \rightarrow \infty$ with $T$ increasing at the same rate as $N$, the random variation in $\widehat{\alpha}_{i}$ vanishes too slowly and the limit distribution of $\widehat{\theta}$ is not centered at $\theta_{0}$; see, e.g., Hahn and Newey (2004).

Early solutions to the IPP were specific for a given model or class of models. For example, an exact solution to the IPP in the sense of restoring fixed- $T$ consistency is possible if a conditional or a marginal likelihood exists that depends on $\theta$ but not on the fixed effects; see, e.g., Cox (1958), Andersen (1970), and Kalbfleisch and Sprott (1970). For many models, however, no such conditional or marginal likelihood exists. Some models (including some semiparametric ones) allow for exact solutions based on differencing, integration, or invariance arguments to eliminate the fixed effects; see, e.g., Anderson and Hsiao (1982), Manski (1987), Arellano and Bond (1991), Honoré (1992), Lancaster (2002), Chamberlain and Moreira (2009), Moreira (2009), and Bonhomme (2012). Again, however, these methods cannot always be applied.

Another approach is to seek more general solutions, which do not depend on the specific functional form of the density. Such solution methods can, in general, only be approximate. They aim at approximate bias correction in some appropriate asymptotic sense. The existing methods vary considerably in terms of approach and ease of implementation. One way of obtaining approximate bias corrections is by model-free methods. For example, Hahn and Newey (2004) and Dhaene and Jochmans (2015) propose jackknife estimators of the bias, Kim and Sun (2016) derive a correction based on the bootstrap, and Arellano and Bonhomme (2009) consider integrated likelihoods with bias-reducing priors. Another approach is to analytically derive the approximate bias of $\widehat{\theta}$, as in Hahn and Newey (2004) and Hahn and Kuersteiner (2011), of the log-likelihood, as in Bester and Hansen (2009) and Arellano and Hahn (2016), or of the score, as in Woutersen (2002). The biascorrected log-likelihood of Arellano and Hahn (2016) is an approximation to an infeasible "target" log-likelihood, in the sense of Pace and Salvan (2006), that is not subject to the IPP. The correction reduces the bias of the log-likelihood (normalized by the number of observations) from $O\left(T^{-1}\right)$ to $O\left(T^{-2}\right)$. The maximizer of the corrected log-likelihood serves as a bias-corrected estimator, inheriting the order of bias $O\left(T^{-2}\right)$ from the corrected log-likelihood.

The analytical bias corrections, i.e., the methods based on an explicit formula for the approximate bias, remove the bias up to an $O\left(T^{-2}\right)$ term. When $T$ is small, this term may still be non-negligible. This paper derives a refined approximation to the infeasible target log-likelihood that removes the $O\left(T^{-2}\right)$ bias term from the log-likelihood as well. The result is a second-order bias-corrected log-likelihood, with bias $O\left(T^{-3}\right)$. The corrected estimator of $\theta_{0}$, obtained from maximizing the corrected log-likelihood, then serves as a second-order bias-corrected estimator, with bias that is expected to be of order $O\left(T^{-3}\right)$. Furthermore, it may be conjectured that the asymptotic distribution of the second-order corrected estimator is correctly centered under asymptotics where $N / T^{5} \rightarrow 0$ (under regularity conditions). By comparison, the condition for the uncorrected estimator $\widehat{\theta}$ to be asymptotically correctly centered is $N / T \rightarrow 0$; and for the first-order corrected estimator the condition is $N / T^{3} \rightarrow 0$.

We derive the second-order corrected log-likelihood by extending the approach of Arellano and 
Hahn (2016). The correction can be applied to a general class of models provided that the data are independent and some regularity conditions are satisfied. The corrected log-likelihood depends only on known quantities such as $Y_{i t}, \widehat{\alpha}_{i}$, and log-likelihood derivatives. Hence, it can be constructed in an automated way from the data.

Given the large $N, T$ approximation arguments used in the various approximate bias correction approaches that currently exist, none of the approaches dominates all others across all models and all sample sizes $N$ and $T$. Some of the general bias correction methods yield fixed- $T$ consistency in some models, essentially as a fortuitous consequence of the special form of the bias of the maximum likelihood estimator or of the profile score. Examples are the jackknife in the many normal means model (Hahn and Newey 2004) and several bias correction methods in the linear autoregressive model with normal errors (Dhaene and Jochmans 2016); for further examples, see Dhaene and Jochmans (2017). However, there are relatively few models where this occurs and the same methods do not, in general, yield fixed- $T$ consistency in other models. Likewise, the remaining bias after approximate bias correction is, in general, highly specific to the method, the model, the sample size ( $T$ in particular), and details of the data generating process such as the true parameter values, the joint distribution of the covariates and the fixed effects, and the initial values (in dynamic models). It may occur, for example, that a given bias correction method yields negligible bias in some circumstances, but much larger bias in other circumstances. In the same vein, second-order bias corrections are not guaranteed, in any specific circumstances, to improve on first-order corrections. Yet, after removing the second-order bias, the remaining bias and the associated distortions for hypothesis tests and confidence intervals decay more quickly in $T$. Therefore, any first-order bias correction with remaining bias $O\left(T^{-2}\right)$ will be dominated by any second-order bias correction for all $T$ sufficiently large. While this may sound as a weak argument in favor of second-order bias corrections (because the required $T$ may be large), in our simulations for logit and probit models we find that the second-order correction dominates the first-order correction for all $T \geq 3$ uniformly over all our designs. Admittedly, simulation results are specific and cannot be generalized without further study, but they nevertheless show that second-order corrections may already effectively improve on first-order corrections for very small $T$, and hence be beneficial in applications. For a related discussion and similar findings on the effect of higher-order versus first-order jackknife bias corrections, see Dhaene and Jochmans (2015).

The paper is organized as follows. Section 2 briefly reviews the IPP and describes the likelihood correction in broad terms. Section 3 gives the main result, the expression of a second-order corrected log-likelihood, and an outline of the derivation. Section 4 presents examples and simulations. Section 5 concludes. Proofs and details of the derivation are given in an appendix.

\section{Incidental parameter problem and likelihood correction}

\subsection{Incidental parameter problem}

Let $Y_{i t}$ denote the $(i, t)$ th observation, where $i=1, \cdots, N$ and $t=1, \cdots, T$. We assume that the $Y_{i t}$ are independent across $i$ and $t$ (conditional on the fixed effects). With $f\left(Y_{i t} ; \theta, \alpha_{i}\right)$ the density of $Y_{i t}, \theta$ the common parameter, and $\alpha_{i}$ the fixed-effect parameter, let

$$
\widehat{\alpha}_{i}(\theta):=\arg \max _{\alpha_{i}} \frac{1}{T} \sum_{t} \log f\left(Y_{i t} ; \theta, \alpha_{i}\right), \quad \alpha_{i}(\theta):=\arg \max _{\alpha_{i}} \frac{1}{T} \sum_{t} \mathbb{E} \log f\left(Y_{i t} ; \theta, \alpha_{i}\right)
$$


be, respectively, the ML estimator of $\alpha_{i}$ and the (infeasible) pseudo-true value of $\alpha_{i}$, for given $\theta$. We use $\mathbb{E}(\cdot)$ to denote the expectation taken under the true density, $f\left(Y_{i t} ; \theta_{0}, \alpha_{i 0}\right)$. Note that $\alpha_{i}\left(\theta_{0}\right)=\alpha_{i 0}$. Now let

$$
\widehat{l}_{i}(\theta):=\frac{1}{T} \sum_{t} \log f\left(Y_{i t} ; \theta, \widehat{\alpha}_{i}(\theta)\right), \quad l_{i}(\theta):=\frac{1}{T} \sum_{t} \log f\left(Y_{i t} ; \theta, \alpha_{i}(\theta)\right)
$$

be, respectively, the profile (or concentrated) log-likelihood and the infeasible "target" log-likelihood, for unit $i$. (Throughout, we normalize all log-likelihood functions by the number of observations.) Averaging $\widehat{l}_{i}(\theta)$ over $i$ and maximizing gives the ML estimator, $\widehat{\theta}$, which for fixed $T$ converges to

$$
\theta_{T}:=\operatorname{plim}_{N \rightarrow \infty} \arg \max _{\theta} \frac{1}{N} \sum_{i} \widehat{l}_{i}(\theta)
$$

There is an IPP when $\theta_{T} \neq \theta_{0}$. When an IPP arises, it is because $\widehat{l}_{i}(\theta)$ uses $\widehat{\alpha}_{i}(\theta)$ for $\alpha_{i}$ in the unprofiled log-likelihood. By contrast, the target log-likelihood, $l_{i}(\theta)$, uses $\alpha_{i}(\theta)$ for $\alpha_{i}$ and is not subject to the IPP. That is,

$$
\theta_{0}=\operatorname{plim}_{N \rightarrow \infty} \arg \max _{\theta} \frac{1}{N} \sum_{i} l_{i}(\theta)
$$

even for fixed $T$. Hence one may view the IPP as resulting from the difference between the profile log-likelihood, $\widehat{l}_{i}(\theta)$, and the target log-likelihood, $l_{i}(\theta)$. The profile $\log$-likelihood is biased due to using $\widehat{\alpha}_{i}(\theta)$ instead of $\alpha_{i}(\theta)$, the bias being $\mathbb{E}\left(\widehat{l}_{i}(\theta)-l_{i}(\theta)\right)=O\left(T^{-1}\right)$.

The IPP is usually understood as fixed- $T$ inconsistency of the ML estimator. In that sense, the IPP becomes less severe as $T$ increases, because

$$
\widehat{\alpha}_{i}(\theta) \stackrel{p}{\rightarrow} \alpha_{i}(\theta), \quad \widehat{l}_{i}(\theta) \stackrel{p}{\rightarrow} l_{i}(\theta), \quad \widehat{\theta} \stackrel{p}{\rightarrow} \theta_{0}, \quad \text { as } T \rightarrow \infty ;
$$

and the large $N, T$ bias of $\widehat{\theta}$ is $\theta_{T}-\theta_{0}=O\left(T^{-1}\right)$. However, the IPP also has a large- $T$ pendant. Hahn and Kuersteiner (2002) and Hahn and Newey (2004) showed that, as $N / T \rightarrow \kappa$ with $0<\kappa<\infty$, the (normal) limit distribution of $\sqrt{N T}\left(\widehat{\theta}-\theta_{0}\right)$ is not centered at 0 , implying asymptotic undercoverage of the usual confidence set centered at $\widehat{\theta}$. The bias in the limit distribution disappears only when $N / T \rightarrow 0$.

The many-normal-means model (Neyman and Scott 1948) is a well-known instance of an IPP. The following example compares the profile and target likelihoods in this model, and their maximizers.

Example 1 (Many normal means) Assume $Y_{i t} \sim \mathcal{N}\left(\alpha_{i 0}, \theta_{0}\right)$, where the mean $\alpha_{i 0}$ may differ across $i$ and the variance $\theta_{0}$ is the same for all $i, t$. The unprofiled log-likelihood, for each $i$, is

$$
\frac{1}{T} \sum_{t} \log f\left(Y_{i t} ; \theta, \alpha_{i}\right)=-\frac{1}{2} \log (2 \pi)-\frac{1}{2} \log \theta-\frac{1}{2 T \theta} \sum_{t}\left(Y_{i t}-\alpha_{i}\right)^{2} .
$$

In this model, $\widehat{\alpha}_{i}(\theta)$ and $\alpha_{i}(\theta)$ are easily obtained in closed form,

$$
\widehat{\alpha}_{i}(\theta)=\bar{Y}_{i .}:=\frac{1}{T} \sum_{t} Y_{i t}, \quad \alpha_{i}(\theta)=\mathbb{E} Y_{i t}=\alpha_{i 0} .
$$


Therefore,

$$
\begin{aligned}
& \widehat{l}_{i}(\theta)=-\frac{1}{2} \log (2 \pi)-\frac{1}{2} \log \theta-\frac{1}{2 T \theta} \sum_{t}\left(Y_{i t}-\bar{Y}_{i .}\right)^{2}, \\
& l_{i}(\theta)=-\frac{1}{2} \log (2 \pi)-\frac{1}{2} \log \theta-\frac{1}{2 T \theta} \sum_{t}\left(Y_{i t}-\alpha_{i 0}\right)^{2} .
\end{aligned}
$$

Maximizing $\frac{1}{N} \sum_{i} \widehat{l}_{i}(\theta)$ gives

$$
\widehat{\theta}=\frac{1}{N T} \sum_{i, t}\left(Y_{i t}-\bar{Y}_{i \cdot}\right)^{2}, \quad \operatorname{plim}_{N \rightarrow \infty} \widehat{\theta}=\theta_{0}-\frac{\theta_{0}}{T} .
$$

Hence, $\widehat{\theta}$ is inconsistent for $\theta_{0}$ when $T$ is fixed. The bias is $O\left(T^{-1}\right)$. On the other hand, maximizing the target log-likelihood $\frac{1}{N} \sum_{i} l_{i}(\theta)$ gives the infeasible estimator

$$
\arg \max _{\theta} \frac{1}{N} \sum_{i} l_{i}(\theta)=\frac{1}{N T} \sum_{i, t}\left(Y_{i t}-\alpha_{i 0}\right)^{2},
$$

which converges in probability to $\theta_{0}$ as $N \rightarrow \infty$ with fixed $T$, hence confirming that the target likelihood is not subject to the IPP.

\subsection{Correcting the likelihood}

The target $\log$-likelihood $l_{i}(\theta)$ is infeasible since $\alpha_{i}(\theta)$ is unknown. One may view $\widehat{l_{i}}(\theta)$ as a feasible approximation to $l_{i}(\theta)$, with $\alpha_{i}(\theta)$ replaced by $\widehat{\alpha}_{i}(\theta)$. However, $\widehat{l}_{i}(\theta)$ is a crude approximation to $l_{i}(\theta)$. It can be refined using quantities which, like $\widehat{l}_{i}(\theta)$, depend only on $\widehat{\alpha}_{i}(\theta)$-not on $\alpha_{i}(\theta)$. Suppose we could construct a $k$ th-order approximating function $\widehat{l}_{i}^{(k)}(\theta)$, independent of $\alpha_{i}(\theta)$, that satisfies

$$
\mathbb{E} l_{i}(\theta)=\mathbb{E} \widehat{l}_{i}^{(k)}(\theta)+O\left(T^{-k-1}\right) .
$$

For example, $\widehat{l}_{i}(\theta)$ corresponds to $k=0$ since $\mathbb{E} l_{i}(\theta)=\mathbb{E} \widehat{l}_{i}(\theta)+O\left(T^{-1}\right)$. Given an approximating function $\widehat{l}_{i}^{(k)}(\theta)$ for some $k \geq 1$, an estimator that is less biased than $\widehat{\theta}$ may then be obtained as

$$
\widehat{\theta}^{(k)}:=\arg \max _{\theta} \frac{1}{N} \sum_{i} \widehat{l}_{i}^{(k)}(\theta)
$$

Arellano and Hahn (2016) provide the approximating function for $k=1$, the first order. It takes the form

$$
\widehat{l}_{i}^{(1)}(\theta)=\widehat{l}_{i}(\theta)+\frac{\widehat{b}_{1 i}(\theta)}{T},
$$

where $\widehat{b}_{1 i}(\theta)$ is a function $b_{1 i}(\theta)$ that depends on $\alpha_{i}(\theta)$, but with $\alpha_{i}(\theta)$ replaced by $\widehat{\alpha}_{i}(\theta)$. (The expression for $b_{1 i}(\theta)$ will be given below as part of our second-order correction.) The term $\widehat{b}_{1 i}(\theta) / T$ is an approximate bias correction term. Arellano and Hahn (2016) also show that $\widehat{\theta}^{(1)}$ derived from $\widehat{l}_{i}^{(1)}(\theta)$ as in $(2.1)$ is biased to the order $O\left(T^{-2}\right)$ only. Thus $\widehat{\theta}^{(1)}$ inherits the order of bias reduction from $\widehat{l}_{i}^{(1)}(\theta)$. Our contribution is to provide

$$
\widehat{l}_{i}^{(2)}(\theta)=\widehat{l}_{i}(\theta)+\frac{\widehat{b}_{1 i}(\theta)}{T}+\frac{\widehat{b}_{2 i}(\theta)}{T^{2}},
$$


where $\widehat{b}_{1 i}(\theta)$ is the same as in Arellano and Hahn (2016) and, similarly, $\widehat{b}_{2 i}(\theta)$ is a function $b_{2 i}(\theta)$ that depends on $\alpha_{i}(\theta)$, but with $\alpha_{i}(\theta)$ replaced by $\widehat{\alpha}_{i}(\theta)$. Thus, the approximation is refined to the second order.

Three likely consequences of the refinement in $(2.2)$ are that (i) $\widehat{\theta}^{(2)}$ inherits the bias order, $O\left(T^{-3}\right)$, from $\widehat{l}_{i}^{(2)}(\theta)$ (just as $\widehat{\theta}$ and $\widehat{\theta}^{(1)}$ inherit the bias order from $\widehat{l}_{i}(\theta)$ and $\widehat{l}_{i}^{(1)}(\theta)$ ); (ii) the asymptotic distribution of $\sqrt{N T}\left(\widehat{\theta}^{(2)}-\theta_{0}\right)$ is normal and centered at 0 under asymptotics where $N / T^{5} \rightarrow 0$ as $N, T \rightarrow \infty$; and (iii) test statistics based on $\widehat{l}_{i}^{(2)}(\theta)$ have an asymptotic $\chi^{2}$ null distribution under the same asymptotics. Here, (ii) and (iii) are the natural continuation of the conditions $N / T \rightarrow 0$ for $\sqrt{N T}\left(\widehat{\theta}-\theta_{0}\right)$ to be asymptotically normal and centered at 0 , and $N / T^{3} \rightarrow 0$ for $\sqrt{N T}\left(\widehat{\theta}^{(1)}-\theta_{0}\right)$ to be asymptotically normal and centered at 0 . That is, higher-order corrections allow $T$ to grow more slowly with $N$ (in practical terms, $T$ may be very small compared to $N$ without causing too much trouble). The focus in this paper is on deriving (2.2). The bias correction property of the corresponding estimator, $\widehat{\theta}^{(2)}$, in particular the conjectures that it inherits the bias order $O\left(T^{-3}\right)$ from $\widehat{l}_{i}^{(2)}(\theta)$ and that its asymptotic distribution is correctly centered when $N / T^{5} \rightarrow 0$, and similarly for the corrected likelihood ratio and Wald statistics, will be explored in simulations. An argument for the expected improved rates of convergence will also be given (see Section 3.3).

Importantly, the bias correction has no effect on the large- $N$, large- $T$ asymptotic variance of the estimator. The reason is simply that the bias correction terms in $(2.2)$ vanish as $T \rightarrow \infty$, so $\widehat{\theta}^{(2)}$ and $\widehat{\theta}$ maximize the same objective function apart from an $O_{p}\left(T^{-1}\right)$ difference. The property that bias correction does not affect the asymptotic variance holds generally for the existing IPP bias correction methods, including the jackknife.

\section{Second-order corrected likelihood}

In this section, we state the main result, the second-order corrected log-likelihood function, and briefly outline the derivation. We also examine $\widehat{\theta}^{(2)}$ under asymptotics where $N, T \rightarrow \infty$ and $N / T^{5} \rightarrow 0$. We make the following assumption.

Assumption 1 (i) $\left(\alpha_{i 0}, Y_{i 1}, \cdots, Y_{i T}\right)$ is i.i.d. across $i$ and, given $\alpha_{i 0}, Y_{i t}$ is i.i.d. across t; (ii) $\left(\theta_{0}, \alpha_{i 0}\right) \in \operatorname{int}(\Theta \times \mathcal{A})$, where $\Theta \subset \mathbb{R}^{k}$ and $\mathcal{A} \subset \mathbb{R}$ are compact; (iii) $\left(\theta_{0}, \alpha_{i 0}\right)$ uniquely maximizes $\mathbb{E} \log f\left(Y_{i t} ; \theta, \alpha_{i}\right)$ over $\Theta \times \mathcal{A}$ and, for every $\theta \in \operatorname{int}(\Theta), \alpha_{i}(\theta)$ uniquely maximizes $\mathbb{E} \log f\left(Y_{i t} ; \theta, \alpha_{i}\right)$ over $\mathcal{A}$ and $\alpha_{i}(\theta) \in \operatorname{int} \mathcal{A}$; (iv) there exist constants $c_{1}$ and $c_{2}$ such that

$$
\begin{aligned}
\left|\frac{\partial^{r_{1}+\ldots+r_{k+1}} \log f\left(Y_{i t} ; \theta, \alpha_{i}\right)}{\partial \theta_{1}^{r_{1}} \cdots \partial \theta_{k}^{r_{k}} \partial \alpha_{i}^{r_{k+1}}}\right| & \leq c_{1}, \quad 0 \leq r_{1}+\cdots+r_{k+1} \leq 6, \\
\left|\frac{\partial^{2} \log f\left(Y_{i t} ; \theta, \alpha_{i}\right)}{\partial \alpha_{i}^{2}}\right| & \geq c_{2}, \quad\left|\operatorname{det} \frac{\partial^{2} \log f\left(Y_{i t} ; \theta, \alpha_{i}\right)}{\partial \eta_{i} \partial \eta_{i}^{\prime}}\right| \geq c_{2},
\end{aligned}
$$

where $\eta_{i}:=\left(\theta^{\prime}, \alpha_{i}\right)^{\prime}$.

Assumption 1(i) is a cross-sectional and serial independence assumption; (ii) assumes compact parameter spaces and restricts $\alpha_{i}$ to be scalar; (iii) is a standard identification condition and ensures that $\alpha_{i}(\theta)$ is well-defined; and (iv) guarantees the existence of moments of log-likelihood derivatives (and products thereof) up to the required order and ensures that the Hessians are bounded away from zero. These assumptions, in particular the boundedness assumptions in (iv), 
are strong, probably stronger than needed. However, they considerably simplify the analysis since they will ensure that the expectations exist wherever they are taken, that the stochastic orders of the remainder terms in the expansions do not increase on taking expectations, and that $b_{1 i}(\theta)$ and $b_{2 i}(\theta)$ are bounded. It would seem that the bound $c_{1}$ in (3.1) can be replaced by function $c_{1}\left(Y_{i t}\right)$ that has sufficiently many moments, and that (3.2) can be relaxed to hold in a neighborhood of $\left(\theta_{0}, \alpha_{i 0}\right)$ for every $i$.

\subsection{Main result}

The corrected likelihood involves sums of products of higher-order log-likelihood derivatives with respect to $\alpha_{i}$, which we denote as follows. Let

$$
\begin{aligned}
l_{\text {rit }}(\theta) & :=\left[\partial_{\alpha_{i}}^{r} \log f\left(Y_{i t} ; \theta, \alpha_{i}\right)\right]_{\alpha_{i}=\alpha_{i}(\theta)}, \\
l_{r i}(\theta) & :=\frac{1}{T} \sum_{t}\left[\partial_{\alpha_{i}}^{r} \log f\left(Y_{i t} ; \theta, \alpha_{i}\right)\right]_{\alpha_{i}=\alpha_{i}(\theta)},
\end{aligned}
$$

where $\partial_{\alpha_{i}}^{r}$ is the $r$ th derivative with respect to $\alpha_{i}$. Further, let $(R, P)$ be a pair of $J \times M$ matrices

$$
R:=\left(\begin{array}{ccc}
r_{11} & \cdots & r_{1 M} \\
\vdots & \ddots & \vdots \\
r_{J 1} & \cdots & r_{J M}
\end{array}\right), \quad P:=\left(\begin{array}{ccc}
p_{11} & \cdots & p_{1 M} \\
\vdots & \ddots & \vdots \\
p_{J 1} & \cdots & p_{J M}
\end{array}\right)
$$

of nonnegative integers $r_{j m}$ and $p_{j m}$ such that, for every $j$ and $m$,

$$
r_{j 1} \geq 1, \quad r_{j m}=0 \Leftrightarrow p_{j m}=0, \quad r_{j m}=0 \Rightarrow r_{j m^{\prime}}=0 \text { for all } m^{\prime}>m .
$$

For any such pair $(R, P)$, let

$$
\mathcal{L}_{i}(R, P, \theta):=\frac{1}{T^{\mathcal{P}(R, P)}} \sum_{\left(t_{1}, \cdots, t_{J}\right)} \prod_{j=1}^{J} \prod_{m=1}^{M}\left[l_{r_{j m} i t}(\theta)\right]^{p_{j m}},
$$

where

$$
\mathcal{P}(R, P):=J-\frac{1}{2} \mathcal{Q}(R, P), \quad \mathcal{Q}(R, P):=\sum_{j=1}^{J} 1\left(\sum_{m=1}^{M} r_{j m}=1 \text { and } \sum_{m=1}^{M} p_{j m}=1\right),
$$

$1(\cdot)$ is the indicator function, and the sum in $\mathcal{L}_{i}(R, P, \theta)$ is over all $J$-tuples $\left(t_{1}, \cdots, t_{J}\right)$ of distinct integers $t_{1}, \cdots, t_{J}$, all between 1 and $T$. Alternatively, we shall write

$$
\mathcal{L}_{\mathcal{P} i}\left(\left(\begin{array}{c}
p_{11}, \cdots, p_{1 M} ; \cdots ; p_{J 1}, \cdots, p_{J M} \\
r_{11}, \cdots, r_{1 M} ; \cdots ; r_{J 1}, \cdots, r_{J M}
\end{array}\right), \theta\right):=\mathcal{L}_{i}(R, P, \theta)
$$

to make $\mathcal{P}$ and the $r_{j m}$ and $p_{j m}$ appear explicitly. Some examples of the notation in (3.6) are

$$
\begin{aligned}
\mathcal{L}_{1 / 2, i}\left(\left(\begin{array}{l}
1 \\
1
\end{array}\right), \theta\right) & =\frac{1}{T^{1 / 2}} \sum_{t} l_{1 i t}(\theta)=T^{1 / 2} l_{1 i}(\theta), \\
\mathcal{L}_{1, i}\left(\left(\begin{array}{l}
1 \\
r
\end{array}\right), \theta\right) & =\frac{1}{T} \sum_{t} l_{\text {rit }}(\theta)=l_{\text {ri }}(\theta), \quad r \geq 2,
\end{aligned}
$$




$$
\begin{aligned}
\mathcal{L}_{1, i}\left(\left(\begin{array}{l}
2 \\
1
\end{array}\right), \theta\right) & =\frac{1}{T} \sum_{t} l_{1 i t}^{2}(\theta), \\
\mathcal{L}_{1, i}\left(\left(\begin{array}{l}
1,1 \\
1, r
\end{array}\right), \theta\right) & =\frac{1}{T} \sum_{t} l_{1 i t}(\theta) l_{\text {rit }}(\theta), \quad r \geq 1, \\
\mathcal{L}_{1, i}\left(\left(\begin{array}{l}
1 ; 1 \\
1 ; 1
\end{array}\right), \theta\right) & =\frac{1}{T} \sum_{t_{1} \neq t_{2}} l_{1 i t_{1}}(\theta) l_{1 i t_{2}}(\theta), \\
\mathcal{L}_{3 / 2, i}\left(\left(\begin{array}{l}
1,0 ; 2,1 \\
1,0 ; 1,3
\end{array}\right), \theta\right) & =\frac{1}{T^{3 / 2}} \sum_{t_{1} \neq t_{2}} l_{1 i t_{1}}(\theta) l_{1 i t_{2}}^{2}(\theta) l_{3 i t_{2}}(\theta) .
\end{aligned}
$$

Whenever we use the notation $\mathcal{L}_{i}(R, P, \theta)$ or its explicit form (3.6), it will be implicitly understood that the pair $(R, P)$ is of the form described in (3.3)-(3.4) for some $J$ and $M$. In addition, it will be implicit without further mentioning that the maximum derivative order involved, $\bar{r}:=$ $\bar{r}(R):=\max _{j, m} r_{j m}$, does not exceed 6. By Assumption 1, this ensures that $\sup _{i, \theta} \mathbb{E}\left|\mathcal{L}_{i}(R, P, \theta)\right|<$ $\infty$ for every $(R, P)$ considered below.

The numbers $\mathcal{Q}:=\mathcal{Q}(R, P)$ and $\mathcal{P}:=\mathcal{P}(R, P)$ have the following interpretation. We say that $\mathcal{L}_{i}(R, P, \theta)$ has a score factor (in essence, $\left.l_{1 i}\right)$ if $R$ and $P$ have at least one row, say the $j$ th, of the form

$$
R_{j}=P_{j}=(1,0, \cdots, 0) .
$$

Thus, $\mathcal{Q}(R, P)$ is the number of score factors in $\mathcal{L}_{i}(R, P, \theta)$. We show in the next subsection that $\mathcal{P}(R, P)$ is the least integer or half-integer that ensures $\mathcal{L}_{i}(R, P, \theta)=O_{p}(1)$ without making functional form assumptions about $f$. Hence, given that $\mathcal{P}=J-\mathcal{Q} / 2$, if there is no score factor, the $J$-fold sum in $\mathcal{L}_{i}(R, P, \theta)$ is $O_{p}\left(T^{J}\right)$; every additional score factor reduces that stochastic order by a factor $T^{-1 / 2}$. This explains the normalizing factor $1 / T^{J-\mathcal{Q} / 2}$ in the definition of $\mathcal{L}_{i}(R, P, \theta)$.

Let $\widehat{l}_{r i}(\theta)$ and $\widehat{\mathcal{L}}_{\mathcal{P} i}(\cdot, \theta)$ be $l_{r i}(\theta)$ and $\mathcal{L}_{\mathcal{P} i}(\cdot, \theta)$ with $\alpha_{i}(\theta)$ replaced by $\widehat{\alpha}_{i}(\theta)$. To condense the notation further, in the remainder of this and the next subsection we omit $\theta$ as a function argument and also omit the index $i$. This is without ambiguity because $\theta$ is kept fixed throughout the analysis and because all log-likelihoods and all correction terms are averages across $i$. Hence we shall write

$$
\begin{array}{lll}
\alpha, l, l_{r}, \mathcal{L}_{\mathcal{P}}(\cdot), b_{1}, b_{2} & \text { instead of } & \alpha_{i}(\theta), l_{i}(\theta), l_{r i}(\theta), \mathcal{L}_{\mathcal{P} i}(\cdot, \theta), b_{1 i}(\theta), b_{2 i}(\theta) ; \\
\widehat{\alpha}, \widehat{l}, \widehat{l}_{r}, \widehat{\mathcal{L}}_{\mathcal{P}}(\cdot), \widehat{b}_{1}, \widehat{b}_{2} \quad \text { instead of } \quad \widehat{\alpha}_{i}(\theta), \widehat{l}_{i}(\theta), \widehat{l}_{r i}(\theta), \widehat{\mathcal{L}}_{\mathcal{P}_{i}}(\cdot, \theta), \widehat{b}_{1 i}(\theta), \widehat{b}_{2 i}(\theta) ;
\end{array}
$$

and $\widehat{l}^{(k)}$ instead of $\widehat{l}_{i}^{(k)}(\theta)$. The main result can now be stated as follows.

Proposition 1 (Second-order corrected log-likelihood) Suppose Assumption 1 holds. Let

$$
\widehat{l}^{(2)}:=\widehat{l}+\frac{\widehat{b}_{1}}{T}+\frac{\widehat{b}_{2}}{T^{2}}
$$

where $\widehat{b}_{1}$ and $\widehat{b}_{2}$ are $b_{1}$ and $b_{2}$ evaluated at $\widehat{\alpha}$ instead of $\alpha$, and

$$
\begin{aligned}
& b_{1}:=\frac{\mathcal{L}_{1}\left(\begin{array}{l}
2 \\
1
\end{array}\right)}{2 l_{2}}, \\
& b_{2}:=\frac{\mathcal{L}_{1}\left(\begin{array}{l}
2,1 \\
1,2
\end{array}\right)}{l_{2}^{2}}-\frac{\mathcal{L}_{1}\left(\begin{array}{l}
1,1 \\
1,2
\end{array}\right)^{2}}{l_{2}^{3}}-\frac{\mathcal{L}_{1}\left(\begin{array}{l}
2 \\
1
\end{array}\right) \mathcal{L}_{1}\left(\begin{array}{l}
2 \\
2
\end{array}\right)}{2 l_{2}^{3}}-\frac{\mathcal{L}_{1}\left(\begin{array}{l}
2 \\
1
\end{array}\right) \mathcal{L}_{1}\left(\begin{array}{l}
1,1 \\
1,3
\end{array}\right)}{2 l_{2}^{3}}-\frac{l_{3} \mathcal{L}_{1}\left(\begin{array}{l}
3 \\
1
\end{array}\right)}{3 l_{2}^{3}}
\end{aligned}
$$




$$
+\frac{l_{4} \mathcal{L}_{1}\left(\begin{array}{l}
2 \\
1
\end{array}\right)^{2}}{8 l_{2}^{4}}+\frac{3 l_{3} \mathcal{L}_{1}\left(\begin{array}{l}
2 \\
1
\end{array}\right) \mathcal{L}_{1}\left(\begin{array}{c}
1,1 \\
1,2
\end{array}\right)}{2 l_{2}^{4}}-\frac{3 l_{3}^{2} \mathcal{L}_{1}\left(\begin{array}{l}
2 \\
1
\end{array}\right)^{2}}{8 l_{2}^{5}} .
$$

Then $\mathbb{E} l=\mathbb{E} \widehat{l}^{(2)}+O\left(T^{-3}\right)$.

The derivation of the bias correction terms $b_{1}$ and $b_{2}$ is given in Section 3.2. The term $b_{1}$ and its estimate $\widehat{b}_{1}$ coincide with the first-order bias correction term of Arellano and Hahn (2016) for static models. The following example is an application of Proposition 1.

Example 2 (Many normal means, continued) In this model we have

$$
\begin{aligned}
l_{1} & =\frac{1}{T \theta} \sum_{t}\left(Y_{i t}-\alpha_{i 0}\right), & l_{2}=-1 / \theta, \quad l_{r}=0 \quad \forall r \geq 3, & \mathcal{L}_{2}\left(\begin{array}{l}
1,1 \\
1,3
\end{array}\right)=0, \\
\mathcal{L}_{1}\left(\begin{array}{l}
2 \\
1
\end{array}\right) & =\frac{1}{T \theta^{2}} \sum_{t}\left(Y_{i t}-\alpha_{i 0}\right)^{2}, & & \mathcal{L}_{1}\left(\begin{array}{l}
2 \\
2
\end{array}\right)=1 / \theta^{2}, \\
\mathcal{L}_{1}\left(\begin{array}{l}
1,1 \\
1,2
\end{array}\right) & =-\frac{1}{T \theta^{2}} \sum_{t}\left(Y_{i t}-\alpha_{i 0}\right), & & \mathcal{L}_{1}\left(\begin{array}{l}
2,1 \\
1,2
\end{array}\right)=-\frac{1}{T \theta^{3}} \sum_{t}\left(Y_{i t}-\alpha_{i 0}\right)^{2} .
\end{aligned}
$$

Using

$$
\frac{\mathcal{L}_{1}\left(\begin{array}{l}
2 \\
1
\end{array}\right)}{l_{2}}=\frac{\mathcal{L}_{1}\left(\begin{array}{l}
2,1 \\
1,2
\end{array}\right)}{l_{2}^{2}}=\frac{\mathcal{L}_{1}\left(\begin{array}{l}
2 \\
1
\end{array}\right) \mathcal{L}_{1}\left(\begin{array}{l}
2 \\
2
\end{array}\right)}{l_{2}^{3}}=-\frac{1}{T \theta} \sum_{t}\left(Y_{i t}-\alpha_{i 0}\right)^{2},
$$

we obtain

$$
\begin{aligned}
b_{1} & =-\frac{1}{2 T \theta} \sum_{t}\left(Y_{i t}-\alpha_{i 0}\right)^{2}, \\
b_{2} & =\frac{\mathcal{L}_{1}\left(\begin{array}{l}
2 \\
1
\end{array}\right)}{2 l_{2}}-\frac{\mathcal{L}_{1}\left(\begin{array}{l}
1,1 \\
1,2
\end{array}\right)}{l_{2}^{3}} \\
& =-\frac{1}{2 T \theta} \sum_{t}\left(Y_{i t}-\alpha_{i 0}\right)^{2}+\frac{1}{T^{2} \theta} \sum_{t, t^{\prime}}\left(Y_{i t}-\alpha_{i 0}\right)\left(Y_{i t^{\prime}}-\alpha_{i 0}\right),
\end{aligned}
$$

and, since $\sum_{t, t^{\prime}}\left(Y_{i t}-\bar{Y}_{i}\right)\left(Y_{i t^{\prime}}-\bar{Y}_{i \cdot}\right)=0$,

$$
\widehat{b}_{1}=\widehat{b}_{2}=-\frac{1}{2 T \theta} \sum_{t}\left(Y_{i t}-\bar{Y}_{i}\right)^{2} .
$$

The second-order corrected log-likelihood follows as

$$
\widehat{l}^{(2)}=-\frac{1}{2} \log (2 \pi)-\frac{1}{2} \log \theta-\frac{1}{2 \theta}\left(\frac{1}{T}+\frac{1}{T^{2}}+\frac{1}{T^{3}}\right) \sum_{t}\left(Y_{i t}-\bar{Y}_{i .}\right)^{2},
$$

with corresponding estimator

$$
\widehat{\theta}^{(2)}=\left(\frac{1}{T}+\frac{1}{T}+\frac{1}{T^{3}}\right) \frac{1}{N} \sum_{i, t}\left(Y_{i t}-\bar{Y}_{i \cdot}\right)^{2}
$$




$$
=\left(1-\frac{1}{T^{3}}\right) \frac{1}{N(T-1)} \sum_{i, t}\left(Y_{i t}-\bar{Y}_{i \cdot}\right)^{2}
$$

As $N \rightarrow \infty$ with $T$ fixed,

$$
\operatorname{plim}_{N \rightarrow \infty} \widehat{\theta}^{(2)}=\theta_{0}-\frac{\theta_{0}}{T^{3}}
$$

so the bias of $\widehat{\theta}^{(2)}$ is $O\left(T^{-3}\right)$.

\subsection{Derivation}

The main steps in the derivation are stochastic expansions of $\widehat{\alpha}-\alpha$ and $\widehat{l}-l$ to the required order, similar those in Arellano and Hahn (2016), followed by an approximation of each term in the expansion of $\widehat{l}-l$.

We first make some preliminary observations on the expectation and the stochastic order of $l_{r}$ and $\mathcal{L}_{\mathcal{P}}(R, P)$. Since $l_{1}$ is the $\alpha_{i}$-score evaluated at the pseudo-true value $\alpha_{i}(\theta)$, it follows from well-known properties of the score function that

$$
l_{1}=O_{p}\left(T^{-1 / 2}\right), \quad \mathbb{E} l_{1}=0 .
$$

On the other hand,

$$
l_{r}=O_{p}(1), \quad \mathbb{E} l_{r} \neq 0 \text { (generically), for } r \geq 2,
$$

where $\mathbb{E} l_{r} \neq 0$ is to be understood in a generic sense; that is, without specific functional form assumptions about $f, \mathbb{E} l_{r}$ is not equal to zero in general. These properties carry over to $\mathcal{L}_{\mathcal{P}}(R, P)$, in part via

$$
l_{1}=\frac{1}{T^{1 / 2}} \mathcal{L}_{1 / 2}\left(\begin{array}{l}
1 \\
1
\end{array}\right), \quad l_{r}=\mathcal{L}_{1}\left(\begin{array}{l}
1 \\
r
\end{array}\right), \quad \text { for } r \geq 2,
$$

and, more generally, via the presence or absence of score factors in $\mathcal{L}_{\mathcal{P}}(R, P)$, i.e., via $\mathcal{Q}(R, P)$.

Lemma 1 Suppose Assumption 1 holds. Then, (i) the expectation of $\mathcal{L}_{\mathcal{P}}(R, P)$ is

$$
\begin{aligned}
& \mathbb{E} \mathcal{L}_{\mathcal{P}}(R, P)=0 \text { if } \mathcal{Q}(R, P) \geq 1, \\
& \mathbb{E} \mathcal{L}_{\mathcal{P}}(R, P) \neq 0 \text { (generically) if } \mathcal{Q}(R, P)=0 ;
\end{aligned}
$$

(ii) $\mathcal{P}(R, P)$ is the least integer or half-integer (generically) that ensures $\mathcal{L}_{\mathcal{P}}(R, P)=O_{p}(1)$.

The next step is to derive a stochastic approximation of $\widehat{\alpha}-\alpha$, and use this to approximate $l$.

Lemma 2 Suppose Assumption 1 holds. Then

$$
\widehat{\alpha}-\alpha=a_{1 / 2}+a_{2 / 2}+a_{3 / 2}+a_{4 / 2}+O_{p}\left(T^{-5 / 2}\right)
$$

where

$$
\begin{aligned}
& a_{1 / 2}:=-\frac{l_{1}}{l_{2}}, \quad a_{2 / 2}:=-\frac{l_{1}^{2} l_{3}}{2 l_{2}^{3}}, \quad a_{3 / 2}:=-\frac{l_{1}^{3} l_{3}^{2}}{2 l_{2}^{5}}+\frac{l_{1}^{3} l_{4}}{6 l_{2}^{4}}, \\
& a_{4 / 2}:=-\frac{5 l_{1}^{4} l_{3}^{3}}{8 l_{2}^{7}}+\frac{5 l_{1}^{4} l_{3} l_{4}}{12 l_{2}^{6}}-\frac{l_{1}^{4} l_{5}}{24 l_{2}^{5}},
\end{aligned}
$$

and $a_{j / 2}=O_{p}\left(T^{-j / 2}\right)$ for $j=1, \cdots, 4$. 
Lemma 3 Suppose Assumption 1 holds. Then

$$
l=\widehat{l}+\underbrace{\frac{l_{1}^{2}}{2 l_{2}}}_{A}+\underbrace{\frac{l_{3} l_{1}^{3}}{6 l_{2}^{3}}}_{B}+\underbrace{\frac{l_{3}^{2} l_{1}^{4}}{8 l_{2}^{5}-\frac{l_{4} l_{1}^{4}}{24 l_{2}^{4}}}}_{C}+\underbrace{\frac{l_{5} l_{1}^{5}}{120 l_{2}^{5}}-\frac{l_{3} l_{4} l_{1}^{5}}{12 l_{2}^{6}}}_{D}+O_{p}\left(T^{-3}\right),
$$

where

$$
\begin{array}{rlrlrl}
A & =O_{p}\left(T^{-1}\right), & B=O_{p}\left(T^{-3 / 2}\right), & C=O_{p}\left(T^{-2}\right), & D & =O_{p}\left(T^{-5 / 2}\right), \\
\mathbb{E} A & =O\left(T^{-1}\right), & \mathbb{E} B=O\left(T^{-2}\right), & \mathbb{E} C=O\left(T^{-2}\right), & \mathbb{E} D=O\left(T^{-3}\right) .
\end{array}
$$

The term $D$ in Lemma 3 can be ignored because its expectation is $O\left(T^{-3}\right)$. The terms $A, B$, and $C$ must be retained. Note that, at this stage, we cannot use plug-in estimates $\widehat{l}_{r}$ for the $l_{r}$ appearing in $A$ to $C$ because, in view of $\widehat{l}_{1}=0$, all three terms would vanish. The next step is to rearrange $A, B$, and $C$ to further separate the orders of magnitude.

Lemma 4 Suppose Assumption 1 holds. Then the terms $A, B$, and $C$ can be written as

$$
\begin{aligned}
& A=\underbrace{\frac{\mathcal{L}_{1}\left(\begin{array}{l}
2 \\
1
\end{array}\right)}{2 T l_{2}}}_{A_{1}}+\underbrace{\frac{\mathcal{L}_{1}\left(\begin{array}{l}
1 ; 1 \\
1 ; 1
\end{array}\right)}{2 T l_{2}}}_{A_{2}} \\
& B=\underbrace{\frac{\mathcal{L}_{5 / 2}\left(\begin{array}{l}
1 ; 2 ; 1 \\
1 ; 1 ; 3
\end{array}\right)}{2 T^{3 / 2} l_{2}^{3}}+\frac{\mathcal{L}_{5 / 2}\left(\begin{array}{l}
1 ; 1 ; 1 ; 1 \\
1 ; 1 ; 1 ; 3
\end{array}\right)}{6 T^{3 / 2} l_{2}^{3}}}_{B_{1}}+\underbrace{\frac{l_{3} \mathcal{L}_{1}\left(\begin{array}{l}
3 \\
1
\end{array}\right)}{6 T^{2} l_{2}^{3}}+\frac{\mathcal{L}_{2}\left(\begin{array}{l}
2,0 ; 1,1 \\
1,0 ; 1,3
\end{array}\right)}{2 T^{2} l_{2}^{3}}}_{B_{2}}+\underbrace{\frac{\mathcal{L}_{2}\left(\begin{array}{l}
1,0 ; 1,0 ; 1,1 \\
1,0 ; 1,0 ; 1,3
\end{array}\right)}{2 T^{2} l_{2}^{3}}+\frac{\mathcal{L}_{3 / 2}\left(\begin{array}{l}
1,0 ; 2,1 \\
1,0 ; 1,3
\end{array}\right)}{2 T^{5 / 2} l_{2}^{3}}}_{B_{3}}, \\
& C=\underbrace{\left(\frac{l_{3}^{2}}{8 l_{2}^{5}}-\frac{l_{4}}{24 l_{2}^{4}}\right) \frac{3 \mathcal{L}_{2}\left(\begin{array}{l}
2 ; 2 \\
1 ; 1
\end{array}\right)}{T^{2}}}_{C_{1}}+\underbrace{\left(\frac{l_{3}^{2}}{8 l_{2}^{5}}-\frac{l_{4}}{24 l_{2}^{4}}\right)\left(\frac{6 \mathcal{L}_{2}\left(\begin{array}{l}
1 ; 1 ; 2 \\
1 ; 1 ; 1
\end{array}\right)}{T^{2}}+\frac{\mathcal{L}_{2}\left(\begin{array}{l}
1 ; 1 ; 1 ; 1 \\
1 ; 1 ; 1 ; 1
\end{array}\right)}{T^{2}}+\frac{4 \mathcal{L}_{3 / 2}\left(\begin{array}{l}
1 ; 3 \\
1 ; 1
\end{array}\right)}{T^{5 / 2}}+\frac{\mathcal{L}_{1}\left(\begin{array}{l}
4 \\
1
\end{array}\right)}{T^{3}}\right)}_{C_{2}},
\end{aligned}
$$

where

$$
\begin{array}{rlrlrl}
A_{1} & =O_{p}\left(T^{-1}\right), & A_{2}=O_{p}\left(T^{-1}\right), & B_{1}=O_{p}\left(T^{-3} / 2\right), & B_{2}=O_{p}\left(T^{-2}\right), \\
\mathbb{E} A_{1} & =O\left(T^{-1}\right), & \mathbb{E} A_{2}=O\left(T^{-2}\right), & \mathbb{E} B_{1}=O\left(T^{-2}\right), & \mathbb{E} B_{2}=O\left(T^{-2}\right), \\
B_{3} & =O_{p}\left(T^{-2}\right), & C_{1}=O_{p}\left(T^{-2}\right), & C_{2}=O_{p}\left(T^{-2}\right), & \\
\mathbb{E} B_{3}=O\left(T^{-3}\right), & \mathbb{E} C_{1}=O\left(T^{-2}\right), & \mathbb{E} C_{2}=O\left(T^{-3}\right) . &
\end{array}
$$

The terms $B_{3}$ and $C_{2}$ have expectation $O\left(T^{-3}\right)$ and can be ignored. We now approximate the remaining terms. A key point is that the terms $A_{2}$ and $B_{1}$ have a score factor and, therefore, have a zero-mean dominant stochastic term, which can be ignored. Let $[Z]$ denote a random variable that is $O_{p}(1)$ and has zero mean.

Lemma 5 Suppose Assumption 1 holds. Then the terms $A_{1}, A_{2}, B_{1}, B_{2}$, and $C_{1}$ can be approximated as

$$
A_{1}=\frac{\widehat{\mathcal{L}}_{1}\left(\begin{array}{l}
2 \\
1
\end{array}\right)}{2 T \widehat{l}_{2}}+\frac{\mathcal{L}_{1}\left(\begin{array}{l}
2,1 \\
1,2
\end{array}\right)}{T^{2} l_{2}^{2}}-\frac{2 \mathcal{L}_{1}\left(\begin{array}{l}
1,1 \\
1,2
\end{array}\right)^{2}}{T^{2} l_{2}^{3}}-\frac{l_{3} \mathcal{L}_{1}\left(\begin{array}{l}
3 \\
1
\end{array}\right)}{2 T^{2} l_{2}^{3}}-\frac{\mathcal{L}_{1}\left(\begin{array}{l}
2 \\
1
\end{array}\right) \mathcal{L}_{1}\left(\begin{array}{l}
1,1 \\
1,3
\end{array}\right)}{T^{2} l_{2}^{3}}-\frac{\mathcal{L}_{1}\left(\begin{array}{l}
2 \\
1
\end{array}\right) \mathcal{L}_{1}\left(\begin{array}{l}
2 \\
2
\end{array}\right)}{2 T^{2} l_{2}^{3}}
$$




$$
\begin{aligned}
& +\frac{l_{4} \mathcal{L}_{1}\left(\begin{array}{l}
2 \\
1
\end{array}\right)^{2}}{4 T^{2} l_{2}^{4}}+\frac{3 l_{3} \mathcal{L}_{1}\left(\begin{array}{l}
2 \\
1
\end{array}\right) \mathcal{L}_{1}\left(\begin{array}{l}
1,1 \\
1,2
\end{array}\right)}{T^{2} l_{2}^{4}}-\frac{3 l_{3}^{2} \mathcal{L}_{1}\left(\begin{array}{l}
2 \\
1
\end{array}\right)^{2}}{4 T^{2} l_{2}^{5}}+\frac{[Z]}{T^{3 / 2}}+O_{p}\left(T^{-3}\right), \\
A_{2}= & \frac{\mathcal{L}_{1}\left(\begin{array}{l}
1,1 \\
1,2
\end{array}\right)^{2}}{T^{2} l_{2}^{3}}+\frac{[Z]}{T^{2}}+O_{p}\left(T^{-3}\right), \\
B_{1}= & -\frac{3 l_{3} \mathcal{L}_{1}\left(\begin{array}{l}
2 \\
1
\end{array}\right) \mathcal{L}_{1}\left(\begin{array}{l}
1,1 \\
1,2
\end{array}\right)}{2 T^{2} l_{2}^{4}}+\frac{[Z]}{T^{3 / 2}}+O_{p}\left(T^{-3}\right), \\
B_{2}= & \frac{l_{3} \mathcal{L}_{1}\left(\begin{array}{l}
3 \\
1
\end{array}\right)}{6 T^{2} l_{2}^{3}}+\frac{\mathcal{L}_{1}\left(\begin{array}{l}
2 \\
1
\end{array}\right) \mathcal{L}_{1}\left(\begin{array}{l}
1,1 \\
1,3
\end{array}\right)}{2 T^{2} l_{2}^{3}}+O_{p}\left(T^{-3}\right), \\
C_{1}= & \frac{3 l_{3}^{2} \mathcal{L}_{1}\left(\begin{array}{l}
2 \\
1
\end{array}\right)^{2}}{8 T^{2} l_{2}^{5}}-\frac{l_{4} \mathcal{L}_{1}\left(\begin{array}{l}
2 \\
1
\end{array}\right)^{2}}{8 T^{2} l_{2}^{4}}+O_{p}\left(T^{-3}\right) .
\end{aligned}
$$

From Lemmas 3 to 5 , it follows that

$$
\begin{aligned}
l & =\widehat{l}+A_{1}+A_{2}+B_{1}+B_{2}+C_{1}+\frac{[Z]}{T^{2}}+O_{p}\left(T^{-3}\right) \\
& =\widehat{l}+\frac{\widehat{\mathcal{L}}_{1}\left(\begin{array}{l}
2 \\
1
\end{array}\right)}{2 T \widehat{l}_{2}}+\frac{b_{2}}{T^{2}}+\frac{[Z]}{T^{3 / 2}}+O_{p}\left(T^{-3}\right) \\
& =\widehat{l}+\frac{\widehat{b}_{1}}{T}+\frac{\widehat{b}_{2}}{T^{2}}+\frac{[Z]}{T^{3 / 2}}+O_{p}\left(T^{-3}\right) \\
& =\widehat{l}^{(2)}+\frac{[Z]}{T^{3 / 2}}+O_{p}\left(T^{-3}\right),
\end{aligned}
$$

which completes the derivation.

\subsection{Asymptotics}

Suppose Assumption 1 holds and consider asymptotics where $N, T \rightarrow \infty$ and $N / T^{5} \rightarrow 0$. The consistency of $\widehat{\theta}^{(2)}$ follows easily. On reintroducing $i$ and $\theta$, we have, by Proposition 1 ,

$$
\sup _{i, \theta}\left|\mathbb{E} \widehat{l}_{i}^{(2)}(\theta)-\mathbb{E} l_{i}(\theta)\right|=O\left(T^{-3}\right) .
$$

Hence $\sup _{\theta}\left|\mathbb{E} \widehat{L}^{(2)}(\theta)-\mathbb{E} L(\theta)\right|=O\left(T^{-3}\right)$, where $L(\theta):=N^{-1} \sum_{i} l_{i}(\theta)$ and $\widehat{L}^{(2)}(\theta):=N^{-1} \sum_{i} \widehat{l}_{i}^{(2)}(\theta)$; note that $\widehat{\theta}^{(2)}=\arg \max _{\theta} \widehat{L}^{(2)}(\theta)$. Further, $\sup _{\theta}|\mathbb{E} L(\theta)-\overline{\mathbb{E}} L(\theta)| \rightarrow 0$, where $\overline{\mathbb{E}}(\cdot)$ is the unconditional expectation (i.e., over $Y_{i t}$ and $\alpha_{i 0}$ ); note that $\theta_{0}=\arg \max _{\theta} \overline{\mathbb{E}} L(\theta)$ and that $\overline{\mathbb{E}} L(\theta)$ is independent of $N$ and $T$. By Assumption $1,\left|\widehat{L}^{(2)}(\theta)\right|$ is uniformly bounded and, hence, $\sup _{\theta} \mid \widehat{L}^{(2)}(\theta)-$ $\mathbb{E} \widehat{L}^{(2)}(\theta) \mid \stackrel{p}{\rightarrow} 0$. Therefore, $\sup _{\theta}\left|\widehat{L}^{(2)}(\theta)-\overline{\mathbb{E}} L(\theta)\right| \stackrel{p}{\rightarrow} 0$ and, by Theorem 2.1 of Newey and McFadden (1994), $\widehat{\theta}^{(2)} \stackrel{p}{\rightarrow} \theta_{0}$. Furthermore, the limit distribution of $\widehat{\theta}^{(2)}$ is normal under weak assumptions. With probability approaching $1, \partial_{\theta} \widehat{L}^{(2)}\left(\widehat{\theta}^{(2)}\right)=0$ and, hence, by a mean value expansion,

$$
0=\partial_{\theta} \widehat{L}^{(2)}\left(\theta_{0}\right)+\partial_{\theta \theta}^{2} \widehat{L}^{(2)}(\bar{\theta})\left(\widehat{\theta}^{(2)}-\theta_{0}\right)
$$

where $\bar{\theta}$ is a point on the line segment joining $\theta_{0}$ and $\widehat{\theta}^{(2)}$. Rearranging gives

$$
\sqrt{N T}\left(\widehat{\theta}^{(2)}-\theta_{0}\right)=-\left[\partial_{\theta \theta}^{2} \widehat{L}^{(2)}(\bar{\theta})\right]^{-1} \sqrt{N T} \partial_{\theta} \widehat{L}^{(2)}\left(\theta_{0}\right),
$$


where

$$
-\left[\partial_{\theta \theta}^{2} \widehat{L}^{(2)}(\bar{\theta})\right]^{-1} \stackrel{p}{\rightarrow} H^{-1}, \quad H:=-\overline{\mathbb{E}} \partial_{\theta \theta}^{2} L\left(\theta_{0}\right),
$$

and

$$
\sqrt{N T} \partial_{\theta} \widehat{L}^{(2)}\left(\theta_{0}\right)=\underbrace{\sqrt{N T}\left(\partial_{\theta} \widehat{L}^{(2)}\left(\theta_{0}\right)-\mathbb{E} \partial_{\theta} \widehat{L}^{(2)}\left(\theta_{0}\right)\right)}_{[A]}+\underbrace{\sqrt{N T} \mathbb{E} \partial_{\theta} \widehat{L}^{(2)}\left(\theta_{0}\right)}_{[B]} .
$$

Here $[A] \stackrel{d}{\rightarrow} \mathcal{N}(0, H)$, so $\sqrt{N T}\left(\widehat{\theta}^{(2)}-\theta_{0}\right) \stackrel{d}{\rightarrow} \mathcal{N}\left(0, H^{-1}\right)$ provided $[B] \stackrel{p}{\rightarrow} 0$. If the score inherits from $\widehat{l}_{i}^{(2)}$ the order of the bias, $O\left(T^{-3}\right)$, uniformly over $i$ and $\theta$, that is, with $\|\cdot\|$ denoting the Euclidean norm, if

$$
\sup _{i, \theta}\left\|\mathbb{E} \partial_{\theta} \widehat{l}_{i}^{(2)}(\theta)-\mathbb{E} \partial_{\theta} l_{i}(\theta)\right\|=O\left(T^{-3}\right),
$$

- compare with (3.15) - then $\sup _{\theta}\left\|\mathbb{E} \partial_{\theta} \widehat{L}^{(2)}(\theta)-\mathbb{E} \partial_{\theta} L(\theta)\right\|=O\left(T^{-3}\right)$ and it follows that $[B]=$ $\sqrt{N T}\left(\mathbb{E} \partial_{\theta} L\left(\theta_{0}\right)+O\left(T^{-3}\right)\right) \rightarrow 0$ since $\mathbb{E} \partial_{\theta} L\left(\theta_{0}\right)=0$ and $N / T^{5} \rightarrow 0$. We have not derived primitive conditions under which the high-level assumption (3.16) holds. Alternatively, instead of assuming (3.16), note that Assumption 1 already implies

$$
\sup _{i, \theta}\left\|\mathbb{E} \partial_{\theta \theta}^{2} \widehat{l}_{i}^{(2)}(\theta)-\mathbb{E} \partial_{\theta \theta}^{2} l_{i}(\theta)\right\|=O\left(T^{-1}\right)
$$

and, hence, $\sup _{\theta}\left\|\mathbb{E} \partial_{\theta \theta}^{2} \widehat{L}^{(2)}(\theta)-\mathbb{E} \partial_{\theta \theta}^{2} L(\theta)\right\|=O\left(T^{-1}\right)$; then, by the Landau-Kolmogorov inequality, for some constant $C_{2}$,

$$
\begin{aligned}
\sup _{\theta}\left\|\mathbb{E} \partial_{\theta} \widehat{L}^{(2)}(\theta)-\mathbb{E} \partial_{\theta} L(\theta)\right\| & \leq C_{2} \sup _{\theta}\left\|\mathbb{E} \widehat{L}^{(2)}(\theta)-\mathbb{E} L(\theta)\right\|_{\theta}^{1 / 2} \sup _{\theta}\left\|\mathbb{E} \partial_{\theta \theta}^{2} \widehat{L}^{(2)}(\theta)-\mathbb{E} \partial_{\theta \theta}^{2} L(\theta)\right\|^{1 / 2} \\
& =O\left(T^{-2}\right),
\end{aligned}
$$

giving $[B] \rightarrow 0$ provided $N / T^{3} \rightarrow 0$. More generally, if (3.17) holds for some derivative order $r \geq 2$ and if $\mathbb{E}(\cdot)$ and $\partial_{\theta \cdots \theta}^{r}(\cdot)$ can be interchanged, then for some constant $C_{r}$,

$$
\begin{aligned}
\sup _{\theta}\left\|\mathbb{E} \partial_{\theta} \widehat{L}^{(2)}(\theta)-\mathbb{E} \partial_{\theta} L(\theta)\right\| & \leq C_{r} \sup _{\theta}\left\|\mathbb{E} \widehat{L}^{(2)}(\theta)-\mathbb{E} L(\theta)\right\|^{1-1 / r} \sup _{\theta}\left\|\mathbb{E} \partial_{\theta \cdots \theta}^{r} \widehat{L}^{(2)}(\theta)-\mathbb{E} \partial_{\theta \cdots \theta}^{r} L(\theta)\right\|^{1 / r} \\
& =O\left(T^{-3+2 / r}\right)
\end{aligned}
$$

and $[B] \rightarrow 0$ provided $N / T^{5-4 / r} \rightarrow 0$. Summarizing, $\sqrt{N T}\left(\widehat{\theta}^{(2)}-\theta_{0}\right)$ is normally distributed under weak assumptions and correctly centered provided $[B] \rightarrow 0$; the latter holds if $N / T^{k} \rightarrow 0$ for some $k$ up to $k=5$ depending on the assumptions.

\section{Simulations}

\subsection{Bias-corrected estimators}

\subsubsection{Logit model}

We first present simulations for the fixed-effect logit model. The natural estimator in this model is the conditional logit ML estimator, which is fixed- $T$ consistent (see, e.g., Andersen 1970, Chamberlain 1980, and Heckman 1981). However, a fixed- $T$ and $\sqrt{N}$-consistent estimator in fixed-effect 
binary-outcome models exists only for the logit model (Chamberlain 2010). Our focus here is on general bias correction methods and we first compare the ML estimator, $\widehat{\theta}$, and the estimators obtained from first- and second-order profile likelihood corrections, $\widehat{\theta}^{(1)}$ and $\widehat{\theta}^{(2)}$. We generated data from the model

$$
Y_{i t}=1\left(X_{i t} \theta_{0}+\alpha_{i 0}+\varepsilon_{i t} \geq 0\right)
$$

where $\varepsilon_{i t}$ is standard-logistically distributed and $X_{i t}$ is a scalar covariate. Throughout, we set $N=1000 ; T=5,10,20 ;$ and $\theta_{0}=0.5,1$. We used three designs to generate $X_{i t}$ and $\alpha_{i 0}$. In Design $1, X_{i t} \sim \mathcal{N}(0,1)$ and $\alpha_{i 0}=0$; in this case the model could be consistently estimated by pooled logit. In Design 2, $X_{i t} \sim \mathcal{N}(0,1)$ and $\alpha_{i 0} \sim \mathcal{N}(0,1 / 16)$; here the model could be consistently estimated by Gaussian-random-effect logit. In Design $3, X_{i t} \sim \mathcal{N}\left(\alpha_{i 0}, 1\right)$ with $\alpha_{i 0} \sim \mathcal{N}(0,1 / 16)$; in this case, due to the correlation between $X_{i t}$ and $\alpha_{i}$, the model has to be estimated either by a fixed-effect method or by Gaussian-correlated-random-effect logit.

Table 1 presents the simulation results, based on 1000 Monte Carlo replications. The three designs yield very similar results. The IPP occurs already in Design 1, that is, it occurs as soon as fixed effects are being estimated, even when their true values are zero. Uniformly across the designs, the ML estimator is heavily biased and the bias is away from zero. When $T=5$, the bias of $\widehat{\theta}$ varies between $25 \%$ and $30 \%$; when $T=20$, it is still around $5 \%$. The bias-corrected estimators $\widehat{\theta}^{(1)}$ and $\widehat{\theta}^{(2)}$ are both very effective in reducing the bias. This holds, in particular, for the second-order bias-corrected estimator, $\widehat{\theta}^{(2)}$. Even when $T=5$, its bias never exceeds $1 \%$; when $T=10$, its bias is already uniformly below $0.5 \%$. A glance at the table also shows that the bias reductions dramatically decrease the root-mean-squared-error (RMSE). The simulations confirm that the biases of $\widehat{\theta}$ and $\widehat{\theta}^{(1)}$ are $O\left(T^{-1}\right)$ and $O\left(T^{-2}\right)$, respectively. For example, when $T$ doubles, the bias of $\widehat{\theta}$ is reduced by a factor roughly equal to $1 / 2$, and that of $\widehat{\theta}^{(1)}$ by a factor $1 / 4$. For $\widehat{\theta}^{(2)}$ the bias is $O\left(T^{-3}\right)$ but this factor, $1 / 8$ as $T$ doubles, is not well visible because the bias is already so small that the Monte Carlo error dominates. We return to this point in the context of the probit model, where the $O\left(T^{-3}\right)$ bias of $\widehat{\theta}^{(2)}$ will be clearly visible in the simulations.

Figure 1 is a graphical examination of the bias corrections to the log-likelihood in the logit model. The profile log-likelihoods are plotted for two data sets, corresponding to $T=5$ and $T=10$, both generated under Design 1 with $\theta_{0}=0.5$ and $N=100,000$. We set $N$ very large to make the sampling variability of all profile log-likelihoods negligibly small. The plotted curves are $N^{-1} \sum_{i} \widehat{l}(\theta)$ (circles), $N^{-1} \sum_{i} \widehat{l}^{(1)}(\theta)$ (triangles), $N^{-1} \sum_{i} \widehat{l}^{(2)}(\theta)$ (squares), and $N^{-1} \sum_{i} l(\theta)$ (asterisks), all computed for $\theta=0.3, \cdots, 0.7$ with a step size of 0.02 , and with vertical lines indicating the maximizers. A comparison of the two graphs shows that when $T$ increases from 5 to $10, N^{-1} \sum_{i} \widehat{l}^{(2)}(\theta)$ converges faster to $N^{-1} \sum_{i} l(\theta)$ than $N^{-1} \sum_{i} \widehat{l}^{(1)}(\theta)$ and $N^{-1} \sum_{i} \widehat{l}(\theta)$ do; and that $N^{-1} \sum_{i} \widehat{l}^{(1)}(\theta)$ converges to $N^{-1} \sum_{i} l(\theta)$ faster than $N^{-1} \sum_{i} \widehat{l}(\theta)$ does. Even with only $T=5$ periods, $N^{-1} \sum_{i} \widehat{l}^{(2)}(\theta)$ is already very accurate, compared to $N^{-1} \sum_{i} \widehat{l}^{(1)}(\theta)$ and $N^{-1} \sum_{i} \widehat{l}(\theta)$, as an approximation to $N^{-1} \sum_{i} l(\theta)$; and the maximizer of $N^{-1} \sum_{i} \widehat{l}^{(2)}(\theta)$ is very close to the maximizer of $N^{-1} \sum_{i} l(\theta)$, which in turn is nearly identical to $\theta_{0}=0.5$. 
Table 1: Simulations for the logit model $(N=1000)$

\begin{tabular}{|c|c|c|c|c|c|c|c|}
\hline & & mean & $\%$ bias & RMSE & mean & $\%$ bias & RMSE \\
\hline \multirow{2}{*}{$\begin{array}{l}\text { Design } 1 \\
T=5\end{array}$} & \multirow{3}{*}{$\begin{array}{l}\widehat{\theta} \\
\widehat{\theta}^{(1)}\end{array}$} & \multicolumn{3}{|c|}{$\theta_{0}=0.5$} & \multicolumn{3}{|c|}{$\theta_{0}=1$} \\
\hline & & 0.632 & $26.3 \%$ & 0.139 & 1.300 & $30.0 \%$ & 0.305 \\
\hline & & 0.538 & $7.6 \%$ & 0.054 & 1.094 & $9.4 \%$ & 0.105 \\
\hline & $\widehat{\theta}^{(2)}$ & 0.503 & $0.6 \%$ & 0.035 & 1.005 & $0.5 \%$ & 0.043 \\
\hline \multirow[t]{3}{*}{$T=10$} & $\widehat{\theta}$ & 0.557 & $11.4 \%$ & 0.063 & 1.123 & $12.3 \%$ & 0.127 \\
\hline & $\widehat{\theta}^{(1)}$ & 0.509 & $1.9 \%$ & 0.025 & 1.020 & $2.0 \%$ & 0.035 \\
\hline & $\widehat{\theta}^{(2)}$ & 0.500 & $0.1 \%$ & 0.023 & 1.000 & $0.0 \%$ & 0.028 \\
\hline \multirow[t]{3}{*}{$T=20$} & $\widehat{\theta}$ & 0.527 & $5.3 \%$ & 0.032 & 1.056 & $5.6 \%$ & 0.059 \\
\hline & $\widehat{\theta}^{(1)}$ & 0.502 & $0.4 \%$ & 0.017 & 1.004 & $0.4 \%$ & 0.019 \\
\hline & $\widehat{\theta}^{(2)}$ & 0.500 & $0.0 \%$ & 0.016 & 0.999 & $-0.1 \%$ & 0.018 \\
\hline \multicolumn{2}{|l|}{ Design 2} & \multicolumn{3}{|c|}{$\theta_{0}=0.5$} & \multicolumn{3}{|c|}{$\theta_{0}=1$} \\
\hline \multirow[t]{3}{*}{$T=5$} & $\widehat{\theta}$ & 0.632 & $26.3 \%$ & 0.139 & 1.301 & $30.1 \%$ & 0.307 \\
\hline & $\widehat{\theta}^{(1)}$ & 0.538 & $7.6 \%$ & 0.054 & 1.095 & $9.5 \%$ & 0.107 \\
\hline & $\widehat{\theta}^{(2)}$ & 0.503 & $0.6 \%$ & 0.036 & 1.005 & $0.5 \%$ & 0.045 \\
\hline \multirow[t]{3}{*}{$T=10$} & $\widehat{\theta}$ & 0.555 & $11.0 \%$ & 0.061 & 1.124 & $12.4 \%$ & 0.129 \\
\hline & $\widehat{\theta}^{(1)}$ & 0.507 & $1.5 \%$ & 0.025 & 1.021 & $2.1 \%$ & 0.036 \\
\hline & $\widehat{\theta}^{(2)}$ & 0.499 & $-0.3 \%$ & 0.024 & 1.001 & $0.1 \%$ & 0.028 \\
\hline \multirow[t]{3}{*}{$T=20$} & $\widehat{\theta}$ & 0.526 & $5.2 \%$ & 0.031 & 1.057 & $5.7 \%$ & 0.060 \\
\hline & $\widehat{\theta}^{(1)}$ & 0.501 & $0.3 \%$ & 0.016 & 1.004 & $0.4 \%$ & 0.020 \\
\hline & $\widehat{\theta}^{(2)}$ & 0.499 & $-0.2 \%$ & 0.016 & 1.000 & $0.0 \%$ & 0.019 \\
\hline \multirow{4}{*}{$\begin{array}{l}\text { Design } 3 \\
T=5\end{array}$} & & \multicolumn{3}{|c|}{$\theta_{0}=0.5$} & \multicolumn{3}{|c|}{$\theta_{0}=1$} \\
\hline & $\widehat{\theta}$ & 0.633 & $26.5 \%$ & 0.140 & 1.295 & $29.5 \%$ & 0.302 \\
\hline & $\widehat{\theta}^{(1)}$ & 0.539 & $7.8 \%$ & 0.054 & 1.090 & $9.0 \%$ & 0.104 \\
\hline & $\widehat{\theta}^{(2)}$ & 0.504 & $0.8 \%$ & 0.035 & 1.000 & $0.0 \%$ & 0.047 \\
\hline \multirow[t]{3}{*}{$T=10$} & $\widehat{\theta}$ & 0.559 & $11.7 \%$ & 0.064 & 1.125 & $12.5 \%$ & 0.129 \\
\hline & $\widehat{\theta}^{(1)}$ & 0.511 & $2.1 \%$ & 0.027 & 1.021 & $2.1 \%$ & 0.035 \\
\hline & $\widehat{\theta}^{(2)}$ & 0.502 & $0.3 \%$ & 0.024 & 1.001 & $0.1 \%$ & 0.028 \\
\hline \multirow[t]{3}{*}{$T=20$} & $\widehat{\theta}$ & 0.528 & $5.6 \%$ & 0.033 & 1.058 & $5.8 \%$ & 0.062 \\
\hline & $\widehat{\theta}^{(1)}$ & 0.503 & $0.7 \%$ & 0.017 & 1.006 & $0.6 \%$ & 0.020 \\
\hline & $\widehat{\theta}^{(2)}$ & 0.501 & $0.2 \%$ & 0.016 & 1.001 & $0.1 \%$ & 0.019 \\
\hline
\end{tabular}

Model: $Y_{i t}=1\left(X_{i t} \theta_{0}+\alpha_{i 0}+\varepsilon_{i t} \geq 0\right), \varepsilon_{i t}$ standard logistic. Design 1: $X_{i t} \sim \mathcal{N}(0,1)$, $\alpha_{i 0}=0$; Design 2: $X_{i t} \sim \mathcal{N}(0,1), \alpha_{i 0} \sim \mathcal{N}(0,1 / 16) ;$ Design 3: $X_{i t} \sim \mathcal{N}\left(\alpha_{i 0}, 1\right)$, $\alpha_{i 0} \sim \mathcal{N}(0,1 / 16) . \widehat{\theta}$ is the ML estimate, $\widehat{\theta}^{(1)}$ is the first-order corrected estimate, $\widehat{\theta}^{(2)}$ is the second-order corrected estimate. 1000 replications. 
Figure 1: Profile log-likelihoods for the logit model $\left(\theta_{0}=0.5\right)$
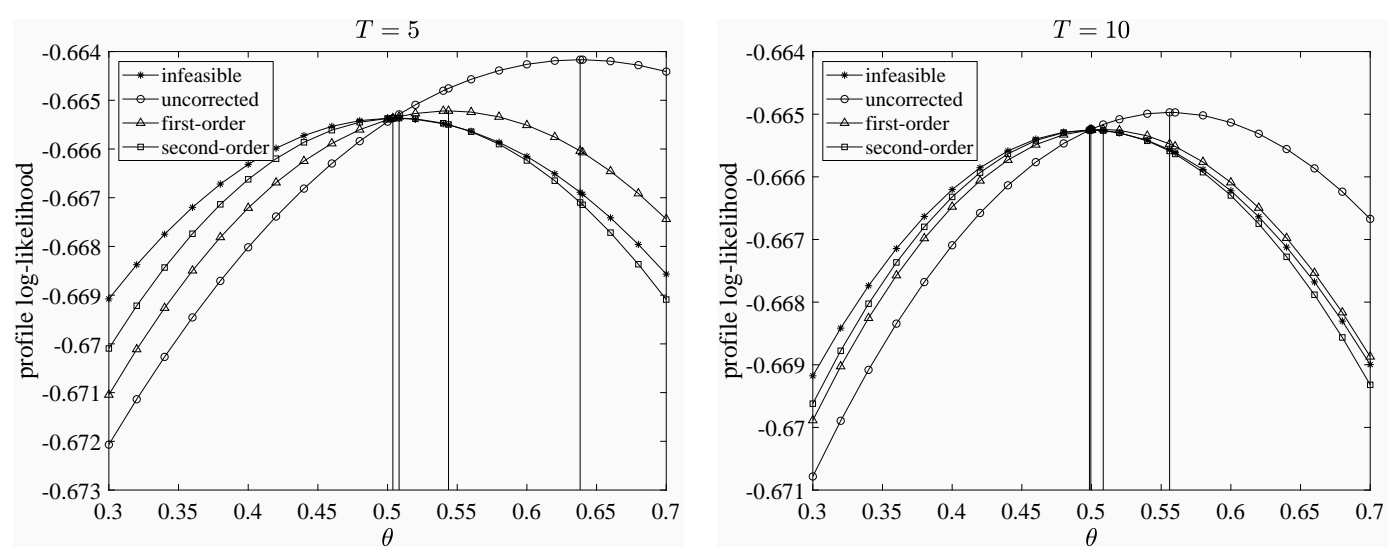

Each figure is computed from a single data set with $N=100,000$. Model: $Y_{i t}=$ $1\left(X_{i t} \theta_{0}+\alpha_{i 0}+\varepsilon_{i t} \geq 0\right), \varepsilon_{i t}$ standard logistic. Data generated under Design 1: $X_{i t} \sim \mathcal{N}(0,1)$, $\alpha_{i 0}=0$. Circles: $\quad N^{-1} \sum_{i} \widehat{l}(\theta)$; triangles: $N^{-1} \sum_{i} \widehat{l}^{(1)}(\theta)$; squares: $\quad N^{-1} \sum_{i} \widehat{l}^{(2)}(\theta)$; asterisks: $N^{-1} \sum_{i} l(\theta)$. All curves are vertically shifted such that they coincide at $\theta_{0}$. Vertical lines at maximizers.

\subsubsection{Probit model}

The next example is the fixed-effect probit model; see also Greene (2004) and Fernández-Val (2009) for simulations in this model. The IPP in the probit model is much harder to resolve than in the logit model because a conditional likelihood does not exist. Furthermore, Chamberlain (2010) showed that fixed- $T$ point identification of $\theta_{0}$ requires unbounded support of the covariates and that, even with unbounded support, fixed- $T$ consistent estimation is not possible at the $\sqrt{N}$ rate. Therefore, one should expect the estimators, in particular the bias-corrected estimators, to perform relatively worse than in the logit model. Table 2 presents simulation results for the probit model under the same designs as for the logit model except that, here, $\varepsilon_{i t} \sim \mathcal{N}(0,1)$. The bias of $\widehat{\theta}$ is between $25 \%$ and $35 \%$ when $T=5$, decreasing to around $5 \%$ when $T=20$. That of $\widehat{\theta}^{(1)}$ is between $12 \%$ and $20 \%$ when $T=5$, decreasing to between $0.5 \%$ and $1 \%$ when $T=20$, roughly at the rate $O\left(T^{-2}\right)$. Lastly, the bias of $\widehat{\theta}^{(2)}$ is between $7 \%$ and $14 \%$ when $T=5$, decreasing to between $0.1 \%$ and $0.2 \%$ when $T=20$, clearly confirming that the bias of $\widehat{\theta}^{(2)}$ decreases at the rate $O\left(T^{-3}\right)$. The conclusion for this model, as for the logit model, is that the bias-corrected estimators considerably improve on the ML estimator. Compared with the logit model, the bias corrections in the probit model are far from perfect when $T$ is very small, yet the remaining bias decreases very rapidly in $T$, in particular when the second-order correction is applied.

Figure 2 presents plots of the profile log-likelihoods for the probit model, paralleling those for the logit model. Again, $N^{-1} \sum_{i} \widehat{l}^{(1)}(\theta)$ provides a closer approximation to the target log-likelihood than $N^{-1} \sum_{i} \widehat{l}(\theta)$ does, and $N^{-1} \sum_{i} \widehat{l}^{(2)}(\theta)$ provides an even closer approximation. The main difference, compared with the logit model, is that the maximizers of $N^{-1} \sum_{i} \widehat{l}^{(1)}(\theta)$ and $N^{-1} \sum_{i} \widehat{l}^{(2)}(\theta)$ are less close to $\theta_{0}=0.5$, in line with the larger biases of $\widehat{\theta}^{(1)}$ and $\widehat{\theta}^{(2)}$ found earlier. 
Table 2: Simulations for the probit model $(N=1000)$

\begin{tabular}{|c|c|c|c|c|c|c|c|}
\hline & & mean & $\%$ bias & $\overline{\mathrm{RMSE}}$ & mean & $\%$ bias & $\overline{\text { RMSE }}$ \\
\hline \multicolumn{2}{|l|}{ Design 1} & \multicolumn{3}{|c|}{$\theta_{0}=0.5$} & \multicolumn{3}{|c|}{$\theta_{0}=1$} \\
\hline \multirow[t]{3}{*}{$T=5$} & $\widehat{\theta}$ & 0.625 & $25.1 \%$ & 0.129 & 1.332 & $33.2 \%$ & 0.336 \\
\hline & $\widehat{\theta}^{(1)}$ & 0.560 & $12.1 \%$ & 0.066 & 1.189 & $18.9 \%$ & 0.194 \\
\hline & $\widehat{\theta}^{(2)}$ & 0.536 & $7.1 \%$ & 0.043 & 1.131 & $13.1 \%$ & 0.138 \\
\hline \multirow[t]{3}{*}{$T=10$} & $\widehat{\theta}$ & 0.550 & $10.0 \%$ & 0.053 & 1.130 & $13.0 \%$ & 0.133 \\
\hline & $\widehat{\theta}^{(1)}$ & 0.512 & $2.4 \%$ & 0.020 & 1.038 & $3.8 \%$ & 0.044 \\
\hline & $\widehat{\theta}^{(2)}$ & 0.503 & $0.6 \%$ & 0.016 & 1.014 & $1.4 \%$ & 0.026 \\
\hline \multirow[t]{3}{*}{$T=20$} & $\widehat{\theta}$ & 0.523 & $4.7 \%$ & 0.026 & 1.058 & $5.8 \%$ & 0.060 \\
\hline & $\widehat{\theta}^{(1)}$ & 0.503 & $0.6 \%$ & 0.011 & 1.008 & $0.8 \%$ & 0.017 \\
\hline & $\widehat{\theta}^{(2)}$ & 0.501 & $0.1 \%$ & 0.011 & 1.001 & $0.1 \%$ & 0.014 \\
\hline \multicolumn{2}{|l|}{ Design 2} & \multicolumn{3}{|c|}{$\theta_{0}=0.5$} & \multicolumn{3}{|c|}{$\theta_{0}=1$} \\
\hline \multirow[t]{3}{*}{$T=5$} & $\widehat{\theta}$ & 0.628 & $25.6 \%$ & 0.132 & 1.333 & $33.3 \%$ & 0.337 \\
\hline & $\widehat{\theta}^{(1)}$ & 0.563 & $12.5 \%$ & 0.068 & 1.190 & $19.0 \%$ & 0.196 \\
\hline & $\widehat{\theta}^{(2)}$ & 0.538 & $7.5 \%$ & 0.046 & 1.132 & $13.2 \%$ & 0.139 \\
\hline \multirow[t]{3}{*}{$T=10$} & $\widehat{\theta}$ & 0.553 & $10.6 \%$ & 0.056 & 1.133 & $13.3 \%$ & 0.135 \\
\hline & $\widehat{\theta}^{(1)}$ & 0.514 & $2.8 \%$ & 0.021 & 1.040 & $4.0 \%$ & 0.046 \\
\hline & $\widehat{\theta}^{(2)}$ & 0.505 & $1.0 \%$ & 0.016 & 1.015 & $1.5 \%$ & 0.026 \\
\hline \multirow[t]{3}{*}{$T=20$} & $\widehat{\theta}$ & 0.524 & $4.8 \%$ & 0.027 & 1.059 & $5.9 \%$ & 0.061 \\
\hline & $\widehat{\theta}^{(1)}$ & 0.503 & $0.7 \%$ & 0.011 & 1.009 & $0.9 \%$ & 0.017 \\
\hline & $\widehat{\theta}^{(2)}$ & 0.501 & $0.2 \%$ & 0.010 & 1.002 & $0.2 \%$ & 0.014 \\
\hline \multicolumn{2}{|l|}{ Design 3} & \multicolumn{3}{|c|}{$\theta_{0}=0.5$} & \multicolumn{3}{|c|}{$\theta_{0}=1$} \\
\hline \multirow[t]{3}{*}{$T=5$} & $\widehat{\theta}$ & 0.630 & $26.1 \%$ & 0.134 & 1.343 & $34.3 \%$ & 0.347 \\
\hline & $\widehat{\theta}^{(1)}$ & 0.565 & $12.9 \%$ & 0.070 & 1.200 & $20.0 \%$ & 0.205 \\
\hline & $\widehat{\theta}^{(2)}$ & 0.540 & $7.9 \%$ & 0.047 & 1.142 & $14.2 \%$ & 0.148 \\
\hline \multirow[t]{3}{*}{$T=10$} & $\widehat{\theta}$ & 0.555 & $10.9 \%$ & 0.057 & 1.138 & $13.8 \%$ & 0.141 \\
\hline & $\widehat{\theta}^{(1)}$ & 0.515 & $3.0 \%$ & 0.022 & 1.044 & $4.4 \%$ & 0.049 \\
\hline & $\widehat{\theta}^{(2)}$ & 0.506 & $1.2 \%$ & 0.016 & 1.018 & $1.8 \%$ & 0.029 \\
\hline \multirow[t]{3}{*}{$T=20$} & $\widehat{\theta}$ & 0.524 & $4.8 \%$ & 0.027 & 1.061 & $6.1 \%$ & 0.063 \\
\hline & $\widehat{\theta}^{(1)}$ & 0.503 & $0.5 \%$ & 0.011 & 1.010 & $1.0 \%$ & 0.018 \\
\hline & $\widehat{\theta}^{(2)}$ & 0.500 & $0.0 \%$ & 0.011 & 1.002 & $0.2 \%$ & 0.015 \\
\hline
\end{tabular}

Model: $Y_{i t}=1\left(X_{i t} \theta_{0}+\alpha_{i 0}+\varepsilon_{i t} \geq 0\right), \varepsilon_{i t} \sim \mathcal{N}(0,1)$. Design 1: $X_{i t} \sim \mathcal{N}(0,1)$, $\alpha_{i 0}=0$; Design 2: $X_{i t} \sim \mathcal{N}(0,1), \alpha_{i 0} \sim \mathcal{N}(0,1 / 16) ;$ Design 3: $X_{i t} \sim \mathcal{N}\left(\alpha_{i 0}, 1\right)$, $\alpha_{i 0} \sim \mathcal{N}(0,1 / 16) . \widehat{\theta}$ is the ML estimate, $\widehat{\theta}^{(1)}$ is the first-order corrected estimate, $\widehat{\theta}^{(2)}$ is the second-order corrected estimate. 1000 replications. 
Figure 2: Profile log-likelihoods for the probit model $\left(\theta_{0}=0.5\right)$
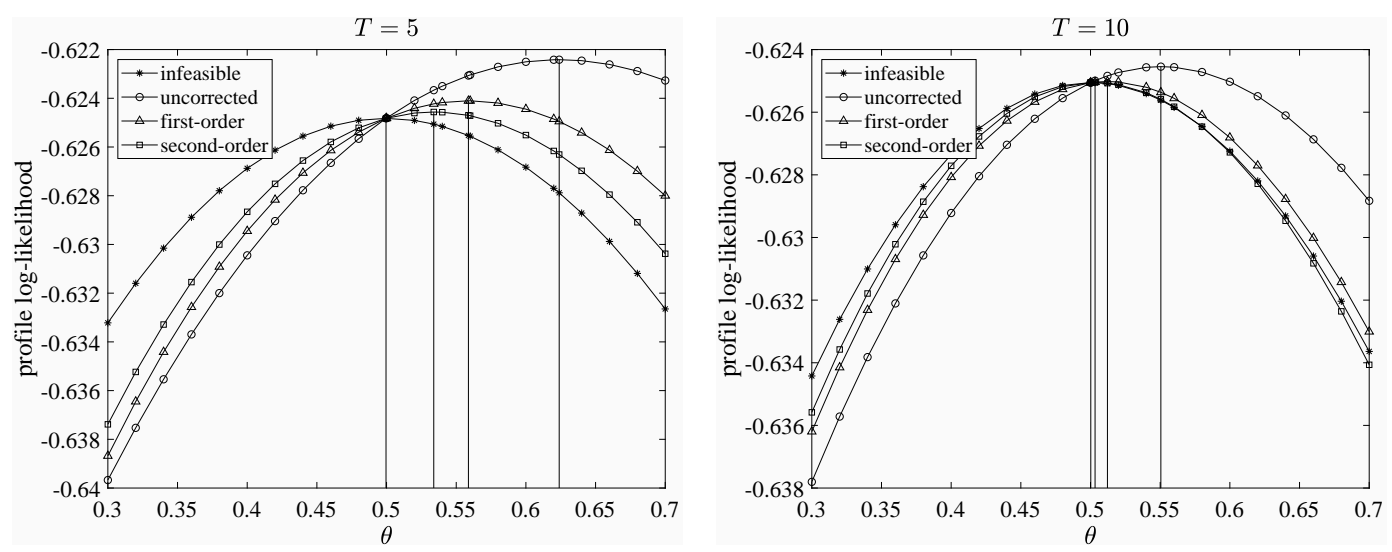

Each figure is computed from a single data set with $N=100,000$. Model: $Y_{i t}=$ $1\left(X_{i t} \theta_{0}+\alpha_{i 0}+\varepsilon_{i t} \geq 0\right), \varepsilon_{i t} \sim \mathcal{N}(0,1)$. Data generated under Design 1: $X_{i t} \sim \mathcal{N}(0,1), \alpha_{i 0}=0$ Circles: $N^{-1} \sum_{i} \widehat{l}(\theta)$; triangles: $N^{-1} \sum_{i} \widehat{l}^{(1)}(\theta)$; squares: $N^{-1} \sum_{i} \widehat{l}^{(2)}(\theta)$; asterisks: $N^{-1} \sum_{i} l(\theta)$. All curves are vertically shifted such that they coincide at $\theta_{0}$. Vertical lines at maximizers.

\subsubsection{Poisson model}

Our last example is the Poisson model, where $Y_{i t}$ is Poisson distributed with mean $\exp \left(X_{i t} \theta_{0}+\alpha_{i 0}\right)$. In this model, there is no IPP (see Lancaster 2002 and Blundell, Griffith, and Windmeijer 2002), so it is of interest to examine the effect of (unnecessary) bias correction. In fact, the bias correction potentially introduces bias, although only of order $O\left(T^{-2}\right)$ and $O\left(T^{-3}\right)$ for $\widehat{\theta}^{(1)}$ and $\widehat{\theta}^{(2)}$, respectively. We generated data $Y_{i t} \sim \operatorname{Poisson}\left(\exp \left(X_{i t} \theta_{0}+\alpha_{i 0}\right)\right)$ with $X_{i t} \sim \mathcal{U}(0,1), \alpha_{i 0}=0, \theta_{0}=0.5$, $N=1000$, and $T=10,20$. Table 3 presents the results, based on 1000 Monte Carlo replications. The simulations confirm the theoretical predictions. The bias correction leaves the bias and the RMSE virtually unchanged.

Table 3: Simulations for the Poisson model $\left(N=1000, \theta_{0}=0.5\right)$

\begin{tabular}{|c|c|c|c|c|c|c|}
\hline & mean & $\%$ bias & RMSE & mean & $\%$ bias & RMSE \\
\hline & \multicolumn{3}{|c|}{$T=10$} & \multicolumn{3}{|c|}{$T=20$} \\
\hline$\widehat{\theta}$ & 0.501 & $0.118 \%$ & 0.032 & 0.501 & $0.117 \%$ & $\begin{array}{l}0.023 \\
\end{array}$ \\
\hline$\widehat{\theta}^{(1)}$ & 0.501 & $0.122 \%$ & 0.032 & 0.501 & $0.115 \%$ & 0.023 \\
\hline$\widehat{\theta}^{(2)}$ & 0.501 & $0.123 \%$ & 0.032 & 0.501 & $0.115 \%$ & 0.023 \\
\hline
\end{tabular}

Model: $Y_{i t} \sim \operatorname{Poisson}\left(\exp \left(X_{i t} \theta_{0}+\alpha_{i 0}\right)\right)$. Data generated with $X_{i t} \sim \mathcal{U}(0,1), \alpha_{i 0}=0 . \widehat{\theta}$ is the ML estimate, $\widehat{\theta}^{(1)}$ is the first-order corrected estimate, $\widehat{\theta}^{(2)}$ is the second-order corrected estimate. 1000 replications.

\subsection{Comparison with the jackknife}

The jackknife is an easy-to-apply method for reducing the IPP bias in panel models. As mentioned, it delivers a fixed- $T$ consistent estimator in the many-normal-means model. More generally, under weak regularity conditions, it reduces the IPP bias from $O\left(T^{-1}\right)$ to $O\left(T^{-2}\right)$, both in its delete-one 
version (Hahn and Newey 2004) and its half-panel version (Dhaene and Jochmans 2015); higherorder versions also exist. Here we briefly compare the analytical bias-correction estimators with the jackknife, using the simulation setup for the fixed-effect probit model under Design 3 (i.e., with $X_{i t} \sim \mathcal{N}\left(\alpha_{i 0}, 1\right)$ and $\left.\alpha_{i 0} \sim \mathcal{N}(0,1 / 16)\right)$ with $\theta_{0}=0.5$. Recall that $\widehat{\theta}^{(1)}$ and $\widehat{\theta}^{(2)}$ still have non-negligible bias when $T=5$ in this setup. We set $T=3, \cdots, 10$ and $N=1000$, and compare with the delete-one jackknife of Hahn and Newey (2004),

$$
\widehat{\theta}_{\mathrm{J}}=T \widehat{\theta}-\frac{T-1}{T} \sum_{t} \widehat{\theta}_{(-t)}
$$

where $\widehat{\theta}_{(-t)}$ is the ML estimator of $\theta_{0}$ obtained from the panel data set with period $t$ deleted, and with the half-panel jackknife of Dhaene and Jochmans (2015),

$$
\widehat{\theta}_{\mathrm{HPJ}}= \begin{cases}2 \widehat{\theta}-\frac{1}{2}\left(\widehat{\theta}_{1: T / 2}+\widehat{\theta}_{T / 2+1: T}\right) & \text { if } T \text { is even } \\ 2 \widehat{\theta}-\frac{1}{2}\left(\frac{\lceil T / 2\rceil \widehat{\theta}_{1:\lceil T / 2\rceil}+\lfloor T / 2\rfloor \widehat{\theta}_{1:\lfloor T / 2\rfloor}}{T}+\frac{\lfloor T / 2\rfloor \widehat{\theta}_{\lceil T / 2\rceil+1: T}+\lceil T / 2\rceil \widehat{\theta}_{\lfloor T / 2\rfloor+1: T}}{T}\right) & \text { if } T \text { is odd }\end{cases}
$$

where $\widehat{\theta}_{t_{1}: t_{2}}$ is the ML estimator of $\theta_{0}$ obtained from the panel data set with periods $t_{1}, \cdots, t_{2}$ only.

\begin{tabular}{|c|c|c|c|c|c|c|c|c|c|c|}
\hline \multirow[b]{2}{*}{$T$} & \multicolumn{5}{|c|}{ mean } & \multicolumn{5}{|c|}{ \% bias } \\
\hline & $\widehat{\theta}$ & $\widehat{\theta}^{(1)}$ & $\widehat{\theta}^{(2)}$ & $\widehat{\theta}_{\mathrm{J}}$ & $\widehat{\theta}_{\mathrm{HPJ}}$ & $\widehat{\theta}$ & $\widehat{\theta}^{(1)}$ & $\widehat{\theta}^{(2)}$ & $\widehat{\theta}_{\mathrm{J}}$ & $\widehat{\theta}_{\text {HPJ }}$ \\
\hline 3 & 0.775 & 0.700 & 0.660 & 0.329 & - & $55.0 \%$ & $39.9 \%$ & $32.0 \%$ & $-34.1 \%$ & - \\
\hline 4 & 0.677 & 0.604 & 0.572 & 0.393 & 0.360 & $35.5 \%$ & $20.8 \%$ & $14.4 \%$ & $-21.4 \%$ & $-28.1 \%$ \\
\hline 5 & 0.630 & 0.564 & 0.539 & 0.436 & 0.400 & $26.0 \%$ & $12.8 \%$ & $7.8 \%$ & $-12.8 \%$ & $-20.1 \%$ \\
\hline 6 & 0.601 & 0.543 & 0.523 & 0.459 & 0.430 & $20.3 \%$ & $8.6 \%$ & $4.6 \%$ & $-8.3 \%$ & $-14.0 \%$ \\
\hline 7 & 0.584 & 0.531 & 0.515 & 0.472 & 0.447 & $16.8 \%$ & $6.3 \%$ & $3.1 \%$ & $-5.6 \%$ & $-10.6 \%$ \\
\hline 8 & 0.570 & 0.523 & 0.510 & 0.479 & 0.462 & $14.1 \%$ & $4.6 \%$ & $2.0 \%$ & $-4.1 \%$ & $-7.6 \%$ \\
\hline 9 & 0.560 & 0.517 & 0.506 & 0.484 & 0.469 & $11.9 \%$ & $3.3 \%$ & $1.2 \%$ & $-3.3 \%$ & $-6.1 \%$ \\
\hline 10 & 0.554 & 0.515 & 0.506 & 0.489 & 0.477 & $10.9 \%$ & $3.0 \%$ & $1.1 \%$ & $-2.3 \%$ & $-4.7 \%$ \\
\hline \multicolumn{6}{|c|}{ RMSE } & & & & & \\
\hline$T$ & $\widehat{\theta}$ & $\widehat{\theta}^{(1)}$ & $\widehat{\theta}^{(2)}$ & $\widehat{\theta}_{\mathrm{J}}$ & $\widehat{\theta}_{\text {HPJ }}$ & & & & & \\
\hline 3 & 0.281 & 0.206 & 0.168 & 0.176 & - & & & & & \\
\hline 4 & 0.182 & 0.110 & 0.080 & 0.110 & 0.151 & & & & & \\
\hline 5 & 0.133 & 0.070 & 0.047 & 0.067 & 0.106 & & & & & \\
\hline 6 & 0.105 & 0.049 & 0.033 & 0.046 & 0.075 & & & & & \\
\hline 7 & 0.087 & 0.038 & 0.025 & 0.033 & 0.057 & & & & & \\
\hline 8 & 0.074 & 0.030 & 0.021 & 0.027 & 0.043 & & & & & \\
\hline 9 & 0.063 & 0.024 & 0.018 & 0.023 & 0.036 & & & & & \\
\hline 10 & 0.057 & 0.022 & 0.017 & 0.019 & 0.029 & & & & & \\
\hline
\end{tabular}

Table 4: Comparison with jackknife estimates (probit model, $N=1000, \theta_{0}=0.5$ )

Model: $Y_{i t}=1\left(X_{i t} \theta_{0}+\alpha_{i 0}+\varepsilon_{i t} \geq 0\right), \varepsilon_{i t} \sim \mathcal{N}(0,1)$. Data generated under Design 3: $X_{i t} \sim$ $\mathcal{N}\left(\alpha_{i 0}, 1\right), \alpha_{i 0} \sim \mathcal{N}(0,1 / 16)$. $\widehat{\theta}$ is the ML estimate, $\widehat{\theta}^{(1)}$ is the first-order corrected estimate, $\widehat{\theta}^{(2)}$ is the second-order corrected estimate, $\widehat{\theta}_{\mathrm{J}}$ is the delete-one jackknife estimate, $\widehat{\theta}_{\mathrm{HPJ}}$ is the half-panel jackknife estimate. 1000 replications. 
Given the independence assumption, the delete-one jackknife is the natural choice of panel jackknife method. The half-panel jackknife, unlike the delete-one jackknife, has the advantage that it can also be applied to dynamic models, but it doubles the magnitude of the $O\left(T^{-2}\right)$ bias term; see Dhaene and Jochmans (2015, p. 1000). Therefore, in the present setting the delete-one jackknife is expected to perform better than the half-panel jackknife.

Table 4 shows the simulation results. All estimators are heavily biased when $T=3,4$ and all biases decrease in $T$ at the predicted rate. The analytical first-order correction, $\widehat{\theta}^{(1)}$, is roughly on par with the delete-one jackknife, both in terms of bias and RMSE, although the jackknife tends to perform slightly better. As predicted, the bias of the half-panel jackknife becomes about twice that of the delete-one jackknife as $T$ increases. Finally, the second-order correction, $\widehat{\theta}^{(2)}$, performs best.

\subsection{Bias-corrected LR and Wald statistics}

Log-likelihood corrections may also be used for improving likelihood-based hypothesis tests. Here we study the effect of the likelihood corrections on the likelihood ratio (LR) and Wald tests using simulations. We have not examined the Lagrange multiplier test but we expect similar improvements as those reported below for the LR and Wald tests.

For the LR test, we compare four versions of the LR statistic: the standard (uncorrected) LR

statistic, based on $\widehat{l}(\theta)$; the first- and second-order corrected LR statistics, based on $\widehat{l}^{(1)}(\theta)$ and $\widehat{l}^{(2)}(\theta)$; and the infeasible LR statistic, based on $l(\theta)$. Given a null hypothesis $H_{0}: \theta_{0}=\theta_{H}$, the standard LR statistic is $L R=-2 \sum_{i} T\left(\widehat{l}_{i}\left(\theta_{H}\right)-\widehat{l}_{i}(\widehat{\theta})\right)$, and similarly for the other LR statistics. For fixed $T$, only the infeasible LR statistic is asymptotically $\chi^{2}$ distributed, as $N \rightarrow \infty$, under the null. All three feasible LR tests are distorted under the null, with rejection probabilities converging to 1 as $N \rightarrow \infty$. For finite $N$, one expects the LR test based on $\widehat{l}(\theta)$ to be the most heavily distorted and that based on $\widehat{l}^{(2)}(\theta)$ to be the least distorted. We ran simulations for the LR statistics in the context of the fixed-effect probit model under Design 3 with $\theta_{0}=0.5$. Figure 3 presents the rejection probabilities of the LR test when the nominal level is $5 \%$, for null hypotheses $H_{0}: \theta_{0}=\theta_{H}$ with $\theta_{H}$ ranging from $\theta_{H}=0.4$ to $\theta_{H}=0.6$. The six plots correspond to $N=100,1000$ and $T=5,10,20$; the rejection probabilities are computed from 10,000 Monte Carlo replications. All theoretical predictions are confirmed by the simulations. The standard LR test heavily overrejects: when $N=100$ and $T=5$, the probability of rejecting $H_{0}: \theta_{0}=0.5$ is $37 \%$; when $T=20$, it is still $11 \%$. For the first-order corrected LR test, these probabilities improve to $19 \%(T=5)$ and $7 \%$ $(T=20)$, and for the second-order corrected test they further improve to $13 \%(T=5)$ and $6 \%$ $(T=20)$. When $N$ is increased to 1000 , the distortions get worse for the standard LR test, with rejection probabilities of nearly $100 \%(T=5)$ and $62 \%(T=20)$. They also get worse, when $N=1000$, for the bias-corrected LR tests, especially when $T=5$, with rejection probabilities of $78 \%$ (first-order correction) and $45 \%$ (second-order correction), but much less so or not at all when $T=20$, with rejection probabilities of $7 \%$ (first-order correction) and $5 \%$ (second-order correction). It is also of interest to consider the power of the various tests, i.e., the rejection probabilities corresponding to incorrect null hypotheses, at least for tests with only mild distortions when the null holds. Here we see, in particular, that for $T \geq 10$ the second-order corrected LR test gives rejection probabilities that are close to those of the infeasible LR statistic, uniformly over the tested values of $\theta_{0}$. The overall conclusion of the simulations for the probit model in this subsection is 
Figure 3: Rejection probability of LR test at $5 \%$ level (probit model, $\theta_{0}=0.5$ )
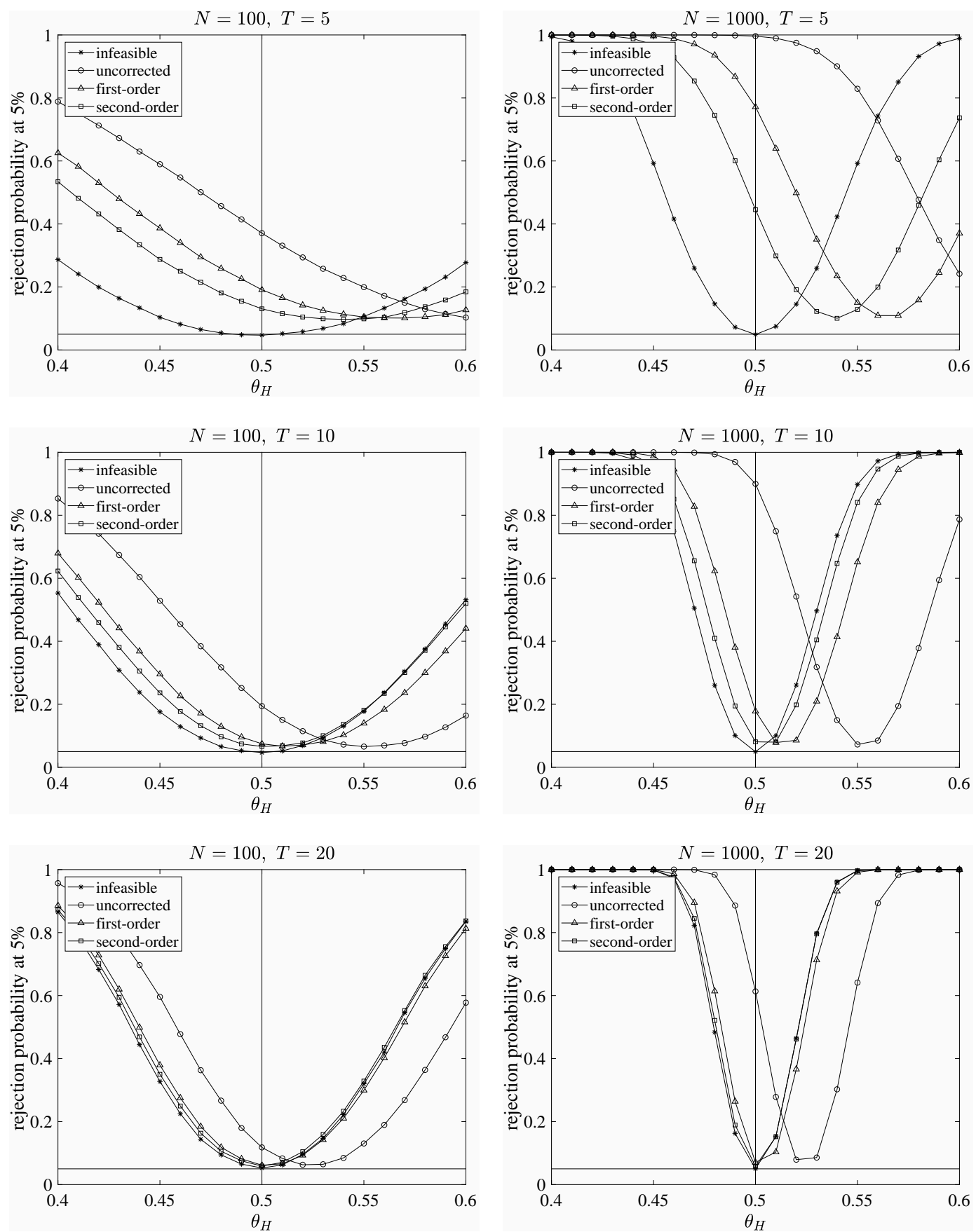

Model: $Y_{i t}=1\left(X_{i t} \theta_{0}+\alpha_{i 0}+\varepsilon_{i t} \geq 0\right), \varepsilon_{i t} \sim \mathcal{N}(0,1)$. Data generated under Design 3: $X_{i t} \sim$ $\mathcal{N}\left(\alpha_{i 0}, 1\right), \alpha_{i 0} \sim \mathcal{N}(0,1 / 16)$. LR tests of $H_{0}: \theta_{0}=\theta_{H}$ based on $\widehat{l}(\theta)$ (circles), $\widehat{l}^{(1)}(\theta)$ (triangles), $\widehat{l}^{(2)}(\theta)$ (squares), and $l(\theta)$ (asterisks), with $\theta_{H}$ on the horizontal axis. Vertical line at $\theta_{0}=0.5$ and horizontal line at 5\%. 10,000 replications. 
Figure 4: Rejection probability of Wald test at $5 \%$ level (probit model, $\theta_{0}=0.5$ )
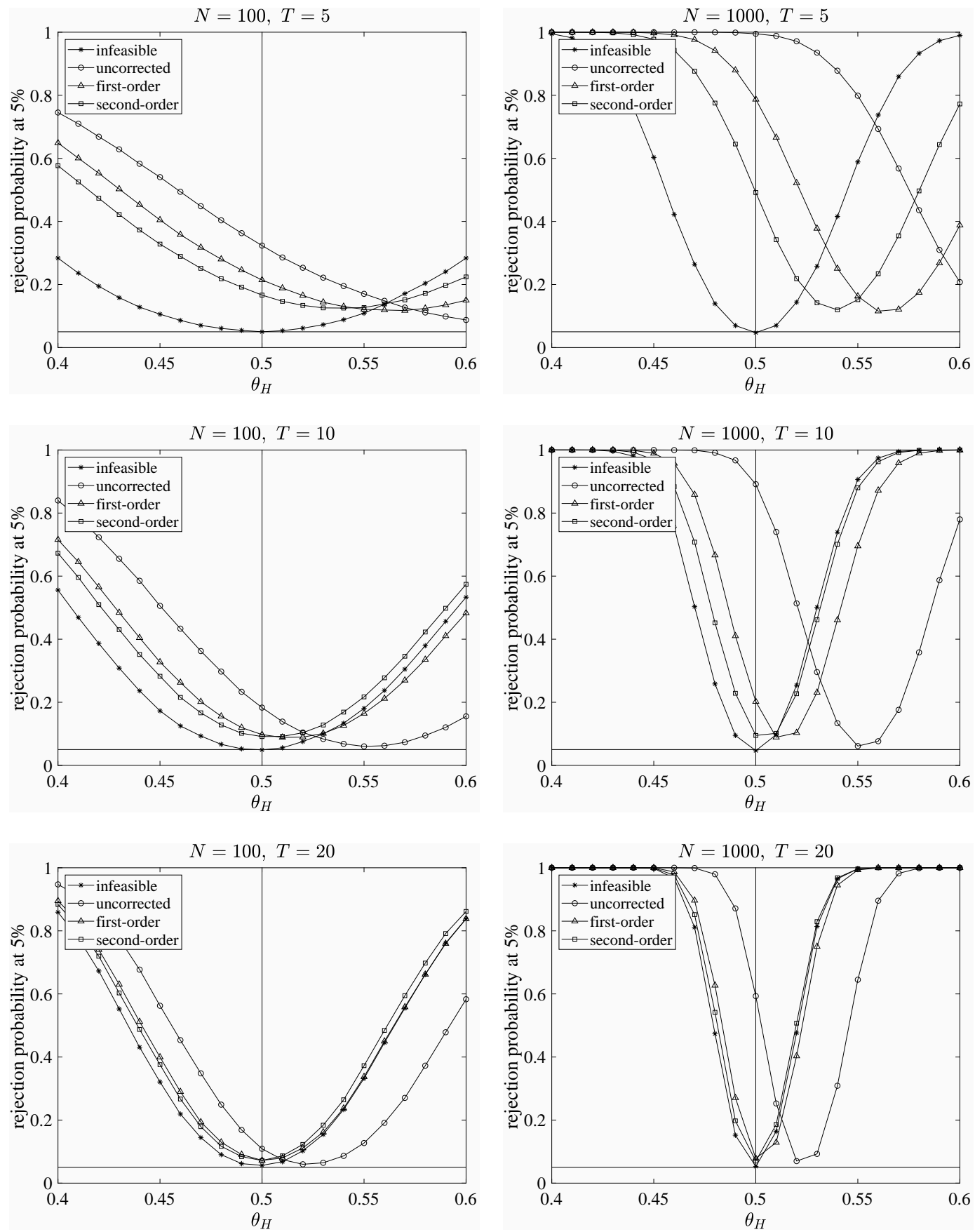

Model: $Y_{i t}=1\left(X_{i t} \theta_{0}+\alpha_{i 0}+\varepsilon_{i t} \geq 0\right), \varepsilon_{i t} \sim \mathcal{N}(0,1)$. Data generated under Design 3: $X_{i t} \sim$ $\mathcal{N}\left(\alpha_{i 0}, 1\right), \alpha_{i 0} \sim \mathcal{N}(0,1 / 16)$. Wald tests of $H_{0}: \theta_{0}=\theta_{H}$ based on $\widehat{l}(\theta)$ (circles), $\widehat{l}^{(1)}(\theta)$ (triangles), $\widehat{l}^{(2)}(\theta)$ (squares), and $l(\theta)$ (asterisks), with $\theta_{H}$ on the horizontal axis. Vertical line at $\theta_{0}=0.5$ and horizontal line at 5\%. 10,000 replications. 
that the LR statistic greatly benefits from bias correction of the profile log-likelihood, and that the second-order correction improves on the first-order correction.

For the Wald test, we compare the analogous four versions of the Wald statistic, based on $\widehat{l}(\theta)$, $\widehat{l}^{(1)}(\theta), \widehat{l}^{(2)}(\theta)$, and the infeasible $l(\theta)$. The one based on $\widehat{l}(\theta)$ is $W=N T\left(\widehat{\theta}-\theta_{H}\right)^{\prime} \widehat{V}^{-1}\left(\widehat{\theta}-\theta_{H}\right)$,

where $\widehat{V}$ is a consistent estimate of the asymptotic variance of the estimator, and similarly for the other Wald statistics. We used the sandwich formula, $\widehat{V}=\widehat{H}^{-1} \widehat{J} \widehat{H}^{-1}$, where, for the ML estimator,

$$
\widehat{H}=-\left(\frac{\partial^{2} \widehat{l}(\theta)}{\partial \theta \partial \theta^{\prime}}\right)_{\theta=\widehat{\theta}}, \quad \widehat{J}=\frac{1}{N(T-1)} \sum_{i, t}\left(\frac{\partial \widehat{l}_{i t}(\theta)}{\partial \theta} \frac{\partial \widehat{l}_{i t}(\theta)}{\partial \theta^{\prime}}\right)_{\theta=\widehat{\theta}}
$$

and analogously for $\widehat{\theta}^{(1)}$ and $\widehat{\theta}^{(2)}$ (with $\widehat{l}^{(1)}$, resp. $\widehat{l}^{(2)}$, replacing $\widehat{l}$ ). For the ML estimator, the degree-of-freedom correction for $\widehat{J}$ is motivated by $\sum_{t}\left(\partial \widehat{l}_{i t}(\theta) / \partial \theta\right)_{\theta=\widehat{\theta}}=0$ (for every $i$ ), and for the bias-corrected estimators by $\sum_{t}\left(\partial \widehat{l}_{i t}^{(j)}(\theta) / \partial \theta\right)_{\theta=\widehat{\theta}^{(j)}} \approx 0, j=1,2$. For the infeasible estimator, $\arg \max _{\theta} l(\theta)$, we use the analogous formulas (with $l$ replacing $\widehat{l}$ ) without degree-of-freedom correction. Figure 4 presents the rejection probabilities of the Wald test using the same simulation setup as for the LR test. The results are very similar to those for the LR test. One difference is that the curves corresponding to $\widehat{l}^{(2)}(\theta)$ and $l(\theta)$ are closer to one another for the LR test than for the Wald test. Another, perhaps more interesting observation is that, for the feasible Wald tests (i.e., based on $\widehat{\theta}, \widehat{\theta}^{(1)}$, or $\hat{\theta}^{(2)}$ ), when $T$ is small, the rejection probability is markedly above $5 \%$ for every value of $\theta_{H}$, an effect that persists as $N$ grows. Hence, it must be the case that, in spite of the degree-of-freedom correction, $\widehat{V}$ still underestimates $V_{T}$, the large- $N$, fixed- $T$ variance of the estimator considered. Thus, there is also an IPP for $V_{T}$. By comparison, the phenomenon that, for small $T$, the rejection probability exceeds $5 \%$ for every value of $\theta_{H}$ also shows up for the LR test, albeit to a somewhat lesser degree.

\section{Concluding remarks}

We derived a second-order bias correction for the profile log-likelihood in nonlinear fixed-effect panel models. The correction removes the first two terms of an expansion of the bias that arises from estimating the fixed effects. As a result, the bias of the profile log-likelihood, normalized by the number of observations, is reduced from $O\left(T^{-1}\right)$ to $O\left(T^{-3}\right)$. Simulations in binary-choice models show that the corresponding maximizer of the corrected log-likelihood inherits the order of bias reduction. The correction can also be applied, without modification, to the more general class of M-estimators, as in Arellano and Hahn (2016).

The bias correction was developed under the assumption of independence of the observations (conditional on covariates and fixed effects). When the observations are dependent, the IPP is typically much more severe. This is already manifest in the linear model, where least-squares is not subject to the IPP under independence, while is it seriously biased in the AR(1) model (Nickell 1981). Therefore, it would be of interest to generalize the second-order correction to the case of dependent data, that is, to extend the analysis of Arellano and Hahn (2016) to the second order.

Given that the second-order bias correction substantially improves on the first-order correction even for small $T$, one may wonder what the third-order and, possibly, arbitrary-order corrections might attain in small- $T$ samples. It should, in principle, be possible to extend the bias correction approach to any order, possibly with the aid of computer-generated expansions. In addition, various other approaches to analytical first-order bias correction, e.g., directly correcting the maximum 
likelihood estimate as in Hahn and Newey (2004) or deriving bias-reducing priors for the integrated likelihood as in Arellano and Bonhomme (2009), could possibly be extended to higher orders, with expected gains similar to those of the profile log-likelihood correction derived here.

Acknowledgements We thank Stéphane Bonhomme, Roger Moon, Cavit Pakel, participants of the 2017 Tinbergen Institute Conference, the 2017 International Panel Data Conference, and the 2018 International Association for Applied Econometrics Annual Conference, two referees, and the editors for helpful comments. Geert Dhaene acknowledges financial support from the Flemish Science Foundation grant G.0505.11.

\section{Appendix: Proofs}

\section{A Preliminaries}

We first present some auxiliary results that are used in the proofs.

Product rule In the derivation we make extensive use of a rule to calculate the product of $\mathcal{L}_{\mathcal{P}(R, P)}(R, P)$ and $\mathcal{L}_{\mathcal{P}(U, V)}(U, V)$ for given $(R, P)$ and $(U, V)$. This requires concatenating $R$ and $U$ in various ways, and similarly for $P$ and $V$. Suppose $R$ is $I \times L$ and $U$ is $J \times M$. Denote the rows of $R$ as $R_{i}$ and those of $U$ as $U_{j}$. Let $C:=\{1, \cdots, I\} \times\{1, \cdots, J\}$ and denote the power set of $C$ as $2^{C}$. Consider the sets $D \in 2^{C}$ that satisfy

$$
(i, j) \in D \Rightarrow\left\{\begin{array}{c}
\left(i, j^{\prime}\right) \notin D \text { for all } j^{\prime} \neq j \\
\left(i^{\prime}, j\right) \notin D \text { for all } i^{\prime} \neq i .
\end{array}\right.
$$

That is, any $D$ is a set of pairs $(i, j) \in C$ where every $i$ is paired at most once, and similarly for every $j$. Let $\mathcal{D}$ be the set of all such $D$. For given $D \in \mathcal{D}$, write $(i, \cdot) \notin D$ to indicate that $(i, j) \notin D$ for every $j$, and similarly for $(\cdot, j) \notin D$. Now let

$$
\begin{aligned}
& {[R, U]_{D}:=\text { a matrix with rows } \begin{cases}\left(R_{i}, U_{j}\right), & (i, j) \in D, \\
\left(R_{i}, 0_{1 \times M}\right), & (i, \cdot) \notin D, \\
\left(U_{j}, 0_{1 \times L}\right), & (\cdot, j) \notin D ;\end{cases} } \\
& {[P, V]_{D}:=\text { a matrix with rows } \begin{cases}\left(P_{i}, V_{j}\right), & (i, j) \in D, \\
\left(P_{i}, 0_{1 \times M}\right), & (i, \cdot) \notin D, \\
\left(V_{j}, 0_{1 \times L}\right), & (\cdot, j) \notin D ;\end{cases} }
\end{aligned}
$$

where, for every $k$, the $k$ th row of $[R, U]_{D}$ and the $k$ th row of $[P, V]_{D}$ are derived from the same index $(i, j) \in D,(i, \cdot) \notin D$, or $(\cdot, j) \notin D$. (That is, the rows of $[R, U]_{D}$ and $[P, V]_{D}$ are constructed in the same order.) The matrices $[R, U]_{D}$ and $[P, V]_{D}$ are $K_{D} \times(L+M)$, where $K_{D}=I+J-|D|$ and $|D|$ denotes the number of elements in $D$. Note that $K_{D} \geq \max \{I, J\}$ since $|D| \leq \min \{I, J\}$. Now, because $\mathcal{L}_{\mathcal{P}(R, P)}(R, P)$ and $\mathcal{L}_{\mathcal{P}(U, V)}(U, V)$ have a multivariate polynomial structure, their product can be rearranged as

$$
\mathcal{L}_{\mathcal{P}(R, P)}(R, P) \mathcal{L}_{\mathcal{P}(U, V)}(U, V)=\sum_{D \in \mathcal{D}} \frac{\mathcal{L}_{\mathcal{P}_{D}}\left([R, U]_{D},[P, V]_{D}\right)}{T^{p_{D}}}
$$


where $\mathcal{P}_{D}:=\mathcal{P}\left([R, U]_{D},[P, V]_{D}\right)$ and $p_{D}$ follows from equating the exponent of $T$ on both sides of $(5.1)$

$$
\mathcal{P}(R, P)+\mathcal{P}(U, V)=\mathcal{P}_{D}+p_{D} .
$$

We refer to (5.1)-(5.2) as the product rule and will apply it many times. In addition, there is an important relationship between $\mathcal{Q}(R, P)$ and $\mathcal{Q}(U, V)$, the number of score factors of the objects on the left of $(5.1)$, and $\mathcal{Q}_{D}:=\mathcal{Q}\left([R, U]_{D},[P, V]_{D}\right)$ and $p_{D}$ associated with the terms on the right. Recall that every score factor, say of $\mathcal{L}_{\mathcal{P}(R, P)}(R, P)$, corresponds to a row of $R$ and $P$, say $R_{i}$ and $P_{i}$, and therefore to an index $i$. Let $\mathcal{I} \subset\{1, \cdots, I\}$ and $\mathcal{J} \subset\{1, \cdots, J\}$ be the index sets corresponding to the score factors of $\mathcal{L}_{\mathcal{P}(R, P)}(R, P)$ and $\mathcal{L}_{\mathcal{P}(U, V)}(U, V)$, and note that $|\mathcal{I}|=\mathcal{Q}(R, P)$ and $|\mathcal{J}|=\mathcal{Q}(U, V)$. Now, from the construction of the matrices $[R, U]_{D}$ and $[P, V]_{D}$, it follows that every score factor in $\mathcal{L}_{\mathcal{P}_{D}}\left([R, U]_{D},[P, V]_{D}\right)$ corresponds to an index $(i, \cdot) \notin D$ with $i \in \mathcal{I}$ or to an index $(\cdot, j) \notin D$ with $j \in \mathcal{J}$; indices $(i, j) \in D$ cannot generate a score factor. Therefore, $\mathcal{Q}_{D}$ is bounded by

$$
\mathcal{Q}(R, P)+\mathcal{Q}(U, V)-2|D| \leq \mathcal{Q}_{D} \leq \mathcal{Q}(R, P)+\mathcal{Q}(U, V) .
$$

The upper bound of $\mathcal{Q}_{D}$ is reached when $(i, \cdot) \notin D$ for every $i \in \mathcal{I}$ and $(\cdot, j) \notin D$ for every $j \in \mathcal{J}$. The lower bound, for given $|D|$, is reached when $i \in \mathcal{I}$ and $j \in \mathcal{J}$ for every $(i, j) \in D$. For large $|D|$, the lower bound in (5.3) may become negative and, therefore, unreachable and uninformative; this happens when $|D|>\left(\mathcal{Q}_{\min }+\mathcal{Q}_{\max }\right) / 2$, where $\mathcal{Q}_{\min }$ and $\mathcal{Q}_{\max }$ are the minimum and the maximum of $\{\mathcal{Q}(R, P), \mathcal{Q}(U, V)\}$. When $\mathcal{Q}_{\max }-\mathcal{Q}_{\min } \geq 2$, an additional, informative lower bound on $\mathcal{Q}_{D}$ is

$$
\mathcal{Q}(R, P)+\mathcal{Q}(U, V)-\mathcal{Q}_{\min }-|D| \leq \mathcal{Q}_{D}, \quad D \in \mathcal{D}: \mathcal{Q}_{\min }<|D|<\mathcal{Q}_{\max },
$$

involving intermediate values of $|D|$. Finally, to make the connection with $p_{D}$, rewrite (5.2) as

$$
I+J-(\mathcal{Q}(R, P)+\mathcal{Q}(U, V)) / 2=K_{D}-\mathcal{Q}_{D} / 2+p_{D}
$$

using the definition of $\mathcal{P}(\cdot)$, and use $K_{D}=I+J-|D|$ to obtain

$$
p_{D}=\mathcal{Q}_{D} / 2-(\mathcal{Q}(R, P)+\mathcal{Q}(U, V)) / 2+|D| .
$$

This, together with the inequalities (5.3), implies $|D| \geq p_{D} \geq 0$. Furthermore, for given $(R, P)$ and $(U, V), p_{D}-\mathcal{Q}_{D} / 2-|D|$ is constant across $D \in \mathcal{D}$.

Simplification rules Useful properties for manipulating and simplifying $\mathcal{L}_{\mathcal{P}}(R, P)$ are: (i) invariance under row permutations, i.e., swapping $\left(R_{j}, P_{j}\right)$ and $\left(R_{j^{\prime}}, P_{j^{\prime}}\right)$; (ii) invariance under column permutations within each row, i.e., swapping $\left(r_{j m}, p_{j m}\right)$ and $\left(r_{j m^{\prime}}, p_{j m^{\prime}}\right)$; (iii) in the explicit notation $\mathcal{L}_{\mathcal{P}}\left(\begin{array}{c}p_{11}, \cdots, p_{1 M} ; \cdots ; p_{J 1}, \cdots, p_{J M} \\ r_{11}, \cdots, r_{1 M} ; \cdots ; r_{J 1}, \cdots, r_{J M}\end{array}\right)$ we may, without ambiguity, (iii.a) drop any zero "column", $\left(\begin{array}{c}p_{j m} \\ r_{j m}\end{array}\right)=\left(\begin{array}{l}0 \\ 0\end{array}\right)$; (iii.b) replace any pair of columns $\left(\begin{array}{c}p_{j m} \\ r_{j m}\end{array}\right),\left(\begin{array}{c}p_{j m^{\prime}} \\ r_{j m^{\prime}}\end{array}\right)$ where $r_{j m}=r_{j m^{\prime}}$ by a single column $\left(\begin{array}{c}p_{j m}+p_{j m^{\prime}} \\ r_{j m}\end{array}\right)$.

Lemma 6 Suppose Assumption 1 holds. If $\mathcal{L}_{\mathcal{P}(R, P)}(R, P)$ has $\mathcal{Q}(R, P)$ score factors and in $\mathcal{L}_{\mathcal{P}(U, V)}(U, V)$ the matrix $U$ is $J \times M$ with $J<\mathcal{Q}(R, P)$, then (i)

$$
\mathbb{E}\left[\mathcal{L}_{\mathcal{P}(R, P)}(R, P) \mathcal{L}_{\mathcal{P}(U, V)}(U, V)\right]=0 ;
$$

(ii) $\mathbb{E}\left[l_{2}^{J} \mathcal{L}_{\mathcal{P}(R, P)}(R, P)\right]=0$. 
Proof Clearly, $\mathcal{Q}(U, V) \leq J$, so $\mathcal{Q}(U, V)<\mathcal{Q}(R, P)$. Further, (5.1) holds with $|D| \leq J$ for every $D \in \mathcal{D}$. When $|D| \leq \mathcal{Q}(U, V)$, (5.3) implies $\mathcal{Q}_{D}>0$. When $\mathcal{Q}(U, V)<|D| \leq J$, (5.4) implies $\mathcal{Q}_{D}>0$. Hence, for every $D \in \mathcal{D}, \mathcal{Q}_{D}>0$. Therefore, every $\mathcal{L}_{\mathcal{P}_{D}}\left([R, U]_{D},[P, V]_{D}\right)$ in (5.1) has zero mean, proving (i). Part (ii) follows on using the product rule iteratively to write $l_{2}^{J}$ as a sum of terms, each of the form $\mathcal{L}_{\mathcal{P}(U, V)}(U, V) / T^{k}$ (for some $k$ ) and satisfying the condition $J<\mathcal{Q}(R, P)$, so that part (i) applies to each term.

Lemma 7 Suppose Assumption 1 holds. If $\mathcal{L}_{\mathcal{P}}(R, P)$ has no score factor, then

$$
\mathcal{L}_{\mathcal{P}}(R, P)=\prod_{j=1}^{J} \mathcal{L}_{1}\left(R_{j}, P_{j}\right)+O_{p}\left(T^{-1}\right),
$$

where $R_{1}, \cdots, R_{J}$ and $P_{1}, \cdots, P_{J}$ are the rows of $R$ and $P$.

Proof Since $\mathcal{L}_{\mathcal{P}}(R, P)$ has no score factor, $\mathcal{P}=J$, so $\mathcal{L}_{\mathcal{P}}(R, P)$ and $\prod_{j=1}^{J} \mathcal{L}_{1}\left(R_{j}, P_{j}\right)$ both depend on $T$ through the factor $T^{-J}$, which ensures both are are $O_{p}(1)$. The difference lies in the summation: $\mathcal{L}_{\mathcal{P}}(R, P)$ involves $\sum_{\left(t_{1}, \cdots, t_{J}\right)}(\cdot)$ where all $t_{1}, \cdots, t_{J}$ are distinct (recall (3.5)) while $\prod_{j=1}^{J} \mathcal{L}_{1}\left(R_{j}, P_{j}\right)$ involves $\sum_{t_{1}, \cdots, t_{J}}(\cdot)$ without the restriction that the $t_{1}, \cdots, t_{J}$ be distinct. The difference, therefore, is $O_{p}\left(T^{-1}\right)$.

Lemma 8 Suppose Assumption 1 holds. Then, (i) for positive integers $p$,

$$
\mathbb{E} l_{1}^{p}= \begin{cases}O\left(T^{-p / 2}\right) & \text { if } p \text { is even } \\ O\left(T^{-(p+1) / 2}\right) & \text { if } p \text { is odd }\end{cases}
$$

(ii) for $r_{1}, \cdots, r_{q} \geq 2$ with $\max _{k} r_{k} \leq 6$,

$$
\mathbb{E}\left(l_{r_{1}} \cdots l_{r_{q}} \mathcal{L}_{\mathcal{P}}(R, P)\right)=O\left(T^{-\mathcal{Q} / 2}\right)
$$

where $\mathcal{Q}$ is the number of score factors of $\mathcal{L}_{\mathcal{P}}(R, P)$; (iii) for positive integers $p$,

$$
\mathbb{E}\left(\frac{l_{r_{1}} \cdots l_{r_{q}} \mathcal{L}_{\mathcal{P}}(R, P)}{l_{2}^{p}}\right)=O\left(T^{-\mathcal{Q} / 2}\right)
$$

Proof Part (i) follows from $l_{1}=Z / T^{1 / 2}+O_{p}\left(T^{-1}\right)$, where $Z$ is normally distributed with zero mean and variance independent of $T$. For part (ii), write $l_{r_{1}}=\mathcal{L}_{1}\left(r_{1}, 1\right)$ and write $l_{r_{1}} \cdots l_{r_{q}} \mathcal{L}_{\mathcal{P}}(R, P)$ simply as $\mathcal{L}$. By the product rule, $l_{r_{1}} \mathcal{L}_{\mathcal{P}}(R, P)$ is of the form

$$
l_{r_{1}} \mathcal{L}_{\mathcal{P}}(R, P)=\sum_{D \in \mathcal{D}} \frac{\mathcal{L}_{\mathcal{P}_{D}}\left(\left[R, r_{1}\right]_{D},[P, 1]_{D}\right)}{T^{p_{D}}}
$$

where $1+\mathcal{P}=\mathcal{P}_{D}+p_{D}$ for every $D \in \mathcal{D}$. Recall that $p_{D} \geq 0$ and, by Lemma $1, \mathbb{E} \mathcal{L}_{\mathcal{P}_{D}}\left(\left[R, r_{1}\right]_{D},[P, 1]_{D}\right)=$ $O(1)$. Let $\mathcal{Q}_{D}$ denote the number of score factors of $\mathcal{L}_{\mathcal{P}_{D}}\left(\left[R, r_{1}\right]_{D},[P, 1]_{D}\right)$. The terms on the righthand side of (5.5) fall into three groups: (i) exactly one term has $\mathcal{P}_{D}=1+\mathcal{P}, p_{D}=0$, and $\mathcal{Q}_{D}=\mathcal{Q}$; (ii) $Q$ terms have $\mathcal{P}_{D}=\mathcal{P}+1 / 2, p_{D}=1 / 2$, and $\mathcal{Q}_{D}=\mathcal{Q}-1$; (iii) all other terms, if any, have 
$\mathcal{P}_{D}=\mathcal{P}, p_{D}=1$, and $\mathcal{Q}_{D}=\mathcal{Q}$. If $\mathcal{Q}=0$, then every term of $l_{r_{1}} \mathcal{L}_{\mathcal{P}}(R, P)$ has mean $O(1)$ or $O\left(T^{-1}\right)$, hence $\mathbb{E}\left(l_{r_{1}} \mathcal{L}_{\mathcal{P}}(R, P)\right)=O(1)$, and iterating the argument gives $\mathbb{E} \mathcal{L}=O(1)$; so (ii) holds when $\mathcal{Q}=0$. If $\mathcal{Q}>0$, then the terms of $l_{r_{1}} \mathcal{L}_{\mathcal{P}}(R, P)$ in groups (i) and (iii) all have mean zero (by Lemma 1), and those in group (ii) have mean $O\left(T^{-1 / 2}\right)$ if $\mathcal{Q}=1$ (since then $\mathcal{Q}_{D}=0$ and $p_{D}=1 / 2$ ) and zero if $\mathcal{Q}>1$; hence, by iterating the argument, $\mathbb{E} \mathcal{L}=O\left(T^{-\mathcal{Q} / 2}\right)$. For part (iii), a mean value expansion of $1 / l_{2}^{p}$ gives

$$
\frac{1}{l_{2}^{p}}=\underbrace{\sum_{k=0}^{\mathcal{Q}-1} \frac{d_{k}}{k !}\left(l_{2}-\mathbb{E} l_{2}\right)^{k}}_{M_{1}}+\underbrace{\frac{d_{\mathcal{Q}}}{\mathcal{Q} !}\left(l_{2}-\mathbb{E} l_{2}\right)^{\mathcal{Q}}}_{M_{2}}
$$

where

$$
\begin{aligned}
d_{k} & := \begin{cases}h_{k}\left(\mathbb{E} l_{2}\right), & k=0, \cdots, \mathcal{Q}-1 ; \\
h_{k}(m), & k=\mathcal{Q} ;\end{cases} \\
h_{k}(z) & :=\frac{d^{k}}{d z^{k}}\left(\frac{1}{z^{p}}\right)=\frac{(-1)^{k}(k+p-1) !}{(p-1) ! z^{k+p}} .
\end{aligned}
$$

and $m$ is a point between $l_{2}$ and $\mathbb{E} l_{2}$. By part (ii), $\mathbb{E}\left(M_{1} \mathcal{L}\right)=O\left(T^{-\mathcal{Q} / 2}\right)$. Also, $\mathbb{E}\left(M_{2} \mathcal{L}\right)=$ $O\left(T^{-\mathcal{Q} / 2}\right)$ since $d_{\mathcal{Q}}$ is bounded by assumption, $\left(l_{2}-\mathbb{E} l_{2}\right)^{\mathcal{Q}}=O_{p}\left(T^{-\mathcal{Q} / 2}\right)$, and $\mathcal{L}=O_{p}(1)$. Hence part (iii) follows.

\section{B Proofs}

Proof of Lemma 1. Let $R_{j}$ and $P_{j}$ be the $j$ th row of $R$ and $P$, and let

$$
s_{i t}\left(R_{j}, P_{j}\right):=\prod_{m=1}^{M}\left[l_{r_{j m} i t}(\theta)\right]^{p_{j m}} .
$$

Further, let

$$
\begin{aligned}
& \mathcal{J}_{0}:=\left\{j \mid R_{j}=P_{j}=(1,0, \cdots, 0)\right\}, \\
& \mathcal{J}_{1}:=\{1, \cdots, J\} \backslash \mathcal{J}_{0} .
\end{aligned}
$$

The score factors in $\mathcal{L}_{\mathcal{P}}(R, P)$ correspond to the $j \in \mathcal{J}_{0}$. Now write $\mathcal{L}_{\mathcal{P}}(R, P)$ as

$$
\begin{aligned}
\mathcal{L}_{\mathcal{P}}(R, P) & =\frac{1}{T^{\mathcal{P}}} \sum_{\left(t_{1}, \ldots, t_{J}\right)} \prod_{j=1}^{J} s_{i t_{j}}\left(R_{j}, P_{j}\right) \\
& =\frac{1}{T^{\mathcal{P}}} \sum_{\left(t_{1}, \cdots, t_{J}\right)}\left(\prod_{j \in \mathcal{J}_{0}} s_{i t_{j}}\left(R_{j}, P_{j}\right)\right)\left(\prod_{j \in \mathcal{J}_{1}} s_{i t_{j}}\left(R_{j}, P_{j}\right)\right),
\end{aligned}
$$

where $\mathcal{P}=\mathcal{P}(R, P)$. Since the $t_{1}, \cdots, t_{J}$ are distinct, by independence,

$$
\mathbb{E} \mathcal{L}_{\mathcal{P}}(R, P)=\frac{1}{T^{\mathcal{P}}} \sum_{\left(t_{1}, \cdots, t_{J}\right)}\left(\prod_{j \in \mathcal{J}_{0}} \mathbb{E} s_{i t_{j}}\left(R_{j}, P_{j}\right)\right)\left(\prod_{j \in \mathcal{J}_{1}} \mathbb{E} s_{i t_{j}}\left(R_{j}, P_{j}\right)\right) .
$$


Here, $\mathbb{E} s_{i t_{j}}\left(R_{j}, P_{j}\right)=0$ if $j \in \mathcal{J}_{0}$ and $\mathbb{E} s_{i t_{j}}\left(R_{j}, P_{j}\right) \neq 0$ (generically) if $j \in \mathcal{J}_{1}$. So $\mathbb{E} \mathcal{L}_{\mathcal{P}}(R, P)=0$ if and only if $\mathcal{L}_{\mathcal{P}}(R, P)$ has at least one score factor. This proves (i). Further, as $T \rightarrow \infty$,

$$
\sum_{t} s_{i t}\left(R_{j}, P_{j}\right)= \begin{cases}O_{p}\left(T^{1 / 2}\right) & \text { if } j \in \mathcal{J}_{0}, \\ O_{p}(T) & \text { if } j \in \mathcal{J}_{1}\end{cases}
$$

and hence, since the number of elements in $\mathcal{J}_{0}$ is $\mathcal{Q}=\mathcal{Q}(R, P)$,

$$
\begin{aligned}
\mathcal{L}_{\mathcal{P}}(R, P) & =\frac{1}{T^{\mathcal{P}}}\left(\prod_{j \in \mathcal{J}_{0}} O_{p}\left(T^{1 / 2}\right)\right)\left(\prod_{j \in \mathcal{J}_{1}} O_{p}(T)\right) \\
& =\frac{1}{T^{\mathcal{P}}} O_{p}\left(T^{\mathcal{Q} / 2}\right) O_{p}\left(T^{J-\mathcal{Q}}\right) \\
& =O_{p}\left(T^{J-\mathcal{Q} / 2-\mathcal{P}}\right) .
\end{aligned}
$$

This proves (ii).

Proof of Lemma 2 As in Cox and Snell (1968), a Taylor expansion of $\widehat{l}_{1}=0$ around $\alpha$ gives

$$
0=l_{1}+l_{2}(\widehat{\alpha}-\alpha)+\frac{l_{3}}{2}(\widehat{\alpha}-\alpha)^{2}+\frac{l_{4}}{6}(\widehat{\alpha}-\alpha)^{3}+\frac{l_{5}}{24}(\widehat{\alpha}-\alpha)^{4}+O_{p}\left(T^{-5 / 2}\right) .
$$

Hence, on rearranging,

$$
\widehat{\alpha}-\alpha=-\frac{l_{1}}{l_{2}}-\frac{l_{3}}{2 l_{2}}(\widehat{\alpha}-\alpha)^{2}-\frac{l_{4}}{6 l_{2}}(\widehat{\alpha}-\alpha)^{3}-\frac{l_{5}}{24 l_{2}}(\widehat{\alpha}-\alpha)^{4}+O_{p}\left(T^{-5 / 2}\right) .
$$

The representation (3.11), with terms satisfying $a_{j / 2}=O_{p}\left(T^{-j / 2}\right)$, can be obtained via a recursive procedure described in Pace and Salvan (1997, Ch. 9). Combining (3.11) and (5.6) gives

$$
\begin{aligned}
a_{1 / 2}+a_{2 / 2}+a_{3 / 2}+a_{4 / 2}= & -\frac{l_{1}}{l_{2}}-\frac{l_{3}}{2 l_{2}}\left(a_{1 / 2}+a_{2 / 2}+a_{3 / 2}+a_{4 / 2}\right)^{2}-\frac{l_{4}}{6 l_{2}}\left(a_{1 / 2}+a_{2 / 2}+a_{3 / 2}+a_{4 / 2}\right)^{3} \\
& -\frac{l_{5}}{24 l_{2}}\left(a_{1 / 2}+a_{2 / 2}+a_{3 / 2}+a_{4 / 2}\right)^{4}+O_{p}\left(T^{-5 / 2}\right) \\
= & -\frac{l_{1}}{l_{2}}-\frac{l_{3}}{2 l_{2}}\left(a_{1 / 2}^{2}+2 a_{1 / 2} a_{2 / 2}+a_{2 / 2}^{2}+2 a_{1 / 2} a_{3 / 2}\right)-\frac{l_{4}}{6 l_{2}}\left(a_{1 / 2}^{3}+3 a_{1 / 2}^{2} a_{2 / 2}\right) \\
& -\frac{l_{5}}{24 l_{2}} a_{1 / 2}^{4}+O_{p}\left(T^{-5 / 2}\right),
\end{aligned}
$$

where terms of small enough stochastic order have been absorbed into the $O_{p}\left(T^{-5 / 2}\right)$ remainder. Using (3.8) and (3.9), grouping terms by their stochastic order gives

$$
\begin{aligned}
& a_{1 / 2}=-\frac{l_{1}}{l_{2}}, \quad a_{2 / 2}=-\frac{l_{3}}{2 l_{2}} a_{1 / 2}^{2}, \quad a_{3 / 2}=-\frac{l_{3}}{l_{2}} a_{1 / 2} a_{2 / 2}-\frac{l_{4}}{6 l_{2}} a_{1 / 2}^{3}, \\
& a_{4 / 2}=-\frac{l_{3}}{2 l_{2}}\left(a_{2 / 2}^{2}+2 a_{1 / 2} a_{3 / 2}\right)-\frac{l_{4}}{2 l_{2}} a_{1 / 2}^{2} a_{2 / 2}-\frac{l_{5}}{24 l_{2}} a_{1 / 2}^{4},
\end{aligned}
$$

and, after a recursive substitution, (3.12). 
Proof of Lemma 3 A Taylor expansion of $\widehat{l}$ around $\alpha$ gives

$$
\widehat{l}=l+l_{1}(\widehat{\alpha}-\alpha)+\frac{l_{2}}{2}(\widehat{\alpha}-\alpha)^{2}+\frac{l_{3}}{6}(\widehat{\alpha}-\alpha)^{3}+\frac{l_{4}}{24}(\widehat{\alpha}-\alpha)^{4}+\frac{l_{5}}{120}(\widehat{\alpha}-\alpha)^{5}+O_{p}\left(T^{-3}\right) .
$$

Hence, by Lemma $\mathbf{2}$,

$$
\begin{aligned}
l & =\widehat{l}-l_{1}(\widehat{\alpha}-\alpha)-\frac{l_{2}}{2}(\widehat{\alpha}-\alpha)^{2}-\frac{l_{3}}{6}(\widehat{\alpha}-\alpha)^{3}-\frac{l_{4}}{24}(\widehat{\alpha}-\alpha)^{4}-\frac{l_{5}}{120}(\widehat{\alpha}-\alpha)^{5}+O_{p}\left(T^{-3}\right) \\
& =\widehat{l}-l_{1} U_{1}-\frac{l_{2} U_{2}}{2}-\frac{l_{3} U_{3}}{6}-\frac{l_{4} U_{4}}{24}-\frac{l_{5} U_{5}}{120}+O_{p}\left(T^{-3}\right),
\end{aligned}
$$

where

$$
\begin{aligned}
& U_{1}=a_{1 / 2}+a_{2 / 2}+a_{3 / 2}+a_{4 / 2}, \\
& U_{2}=a_{1 / 2}^{2}+2 a_{1 / 2} a_{2 / 2}+a_{2 / 2}^{2}+2 a_{1 / 2} a_{3 / 2}+2 a_{1 / 2} a_{4 / 2}+2 a_{2 / 2} a_{3 / 2}, \\
& U_{3}=a_{1 / 2}^{3}+3 a_{1 / 2}^{2} a_{2 / 2}+3 a_{1 / 2}^{2} a_{3 / 2}, \quad U_{4}=a_{1 / 2}^{4}+4 a_{1 / 2}^{3} a_{2 / 2}, \quad U_{5}=a_{1 / 2}^{5} .
\end{aligned}
$$

From (3.12)-(3.13),

$$
\begin{aligned}
& U_{1}=-\frac{l_{1}}{l_{2}}-\frac{l_{1}^{2} l_{3}}{2 l_{2}^{3}}-\frac{l_{1}^{3} l_{3}^{2}}{2 l_{2}^{5}}+\frac{l_{1}^{3} l_{4}}{6 l_{2}^{4}}-\frac{5 l_{1}^{4} l_{3}^{3}}{8 l_{2}^{7}}+\frac{5 l_{1}^{4} l_{3} l_{4}}{12 l_{2}^{6}}-\frac{l_{1}^{4} l_{5}}{24 l_{2}^{5}}, \\
& U_{2}=\frac{l_{1}^{2}}{l_{2}^{2}}+\frac{l_{1}^{3} l_{3}}{l_{2}^{4}}+\frac{5 l_{1}^{4} l_{3}^{2}}{4 l_{2}^{6}}-\frac{l_{1}^{4} l_{4}}{3 l_{2}^{5}}+\frac{l_{1}^{5} l_{5}}{12 l_{2}^{6}}-\frac{l_{1}^{5} l_{3} l_{4}}{l_{2}^{7}}+\frac{7 l_{1}^{5} l_{3}^{3}}{4 l_{2}^{8}}, \\
& U_{3}=-\frac{l_{1}^{3}}{l_{2}^{3}}-\frac{3 l_{1}^{4} l_{3}}{2 l_{2}^{5}}-\frac{3 l_{1}^{5} l_{3}^{2}}{2 l_{2}^{7}}+\frac{l_{1}^{5} l_{4}}{2 l_{2}^{6}}, \quad U_{4}=\frac{l_{1}^{4}}{l_{2}^{4}}+\frac{2 l_{1}^{5} l_{3}}{l_{2}^{6}}, \quad U_{5}=-\frac{l_{1}^{5}}{l_{2}^{5}} .
\end{aligned}
$$

Now (3.14) follows from (5.7) and straightforward algebra. The orders of $A$ to $D$ follow from (3.8)(3.9). By Lemma 8, for each term the order of the expectation is inherited from that of its factor $l_{1}^{p}$, that is, $\mathbb{E} l_{1}^{p}=O\left(T^{-\lceil(p+1) / 2\rceil}\right)$, giving $\mathbb{E}\left(l_{1}^{2} / l_{2}\right)=O\left(\mathbb{E} l_{1}^{2}\right)=T^{-1}, \mathbb{E}\left(l_{3} l_{1}^{3} / l_{2}^{3}\right)=O\left(\mathbb{E} l_{1}^{3}\right)=T^{-2}$, and so on.

Proof of Lemma 4 By (3.10) and the product rule given in (5.1), we have

$$
l_{1}^{2}=\frac{\mathcal{L}_{1 / 2}\left(\begin{array}{l}
1 \\
1
\end{array}\right)^{2}}{T}=\frac{\mathcal{L}_{1}\left(\begin{array}{l}
2 \\
1
\end{array}\right)}{T}+\frac{\mathcal{L}_{1}\left(\begin{array}{l}
1 ; 1 \\
1 ; 1
\end{array}\right)}{T},
$$

which gives the expression for $A$. Further, recursively applying the product rule gives

$$
\begin{aligned}
l_{1}^{3} & =\frac{\mathcal{L}_{1 / 2}\left(\begin{array}{l}
1 \\
1
\end{array}\right)}{T^{1 / 2}}\left(\frac{\mathcal{L}_{1}\left(\begin{array}{l}
2 \\
1
\end{array}\right)}{T}+\frac{\mathcal{L}_{1}\left(\begin{array}{l}
1 ; 1 \\
1 ; 1
\end{array}\right)}{T}\right) \\
& =\frac{\mathcal{L}_{1}\left(\begin{array}{l}
3 \\
1
\end{array}\right)}{T^{2}}+\frac{3 \mathcal{L}_{3 / 2}\left(\begin{array}{l}
1 ; 2 \\
1 ; 1
\end{array}\right)}{T^{3 / 2}}+\frac{\mathcal{L}_{3 / 2}\left(\begin{array}{l}
1 ; 1 ; 1 \\
1 ; 1 ; 1
\end{array}\right)}{T^{3 / 2}} \\
l_{3} l_{1}^{3} & =\frac{l_{3} \mathcal{L}_{1}\left(\begin{array}{l}
3 \\
1
\end{array}\right)}{T^{2}}+\mathcal{L}_{1}\left(\begin{array}{l}
1 \\
3
\end{array}\right)\left(\frac{3 \mathcal{L}_{3 / 2}\left(\begin{array}{l}
1 ; 2 \\
1 ; 1
\end{array}\right)}{T^{3 / 2}}+\frac{\mathcal{L}_{3 / 2}\left(\begin{array}{l}
1 ; 1 ; 1 ; \\
1 ; 1 ;
\end{array}\right)}{T^{3 / 2}}\right) \\
& =\frac{3 \mathcal{L}_{5 / 2}\left(\begin{array}{l}
1 ; 2 ; 1 \\
1 ; 1 ; 3
\end{array}\right)}{T^{3 / 2}}+\frac{\mathcal{L}_{5 / 2}\left(\begin{array}{l}
1 ; 1 ; 1 ; 1 ; 1 \\
1 ; 1 ; 1 ; 3
\end{array}\right)}{T^{3 / 2}}+\frac{l_{3} \mathcal{L}_{1}\left(\begin{array}{l}
3 \\
1
\end{array}\right)}{T^{2}}+\frac{3 \mathcal{L}_{2}\left(\begin{array}{l}
2 ; 1,1 \\
1 ; 1,3
\end{array}\right)}{T^{2}}+\frac{3 \mathcal{L}_{2}\left(\begin{array}{l}
1 ; 1 ; 1 ; 1,1 \\
1 ; 1 ; 3
\end{array}\right)}{T^{2}}+\frac{3 \mathcal{L}_{3 / 2}\left(\begin{array}{l}
1 ; 2,1 \\
1 ; 1 ; 3
\end{array}\right)}{T^{5 / 2}}
\end{aligned}
$$


and the expression for $B$ follows. From (5.8),

$$
l_{1}^{4}=\frac{3 \mathcal{L}_{2}\left(\begin{array}{l}
2 ; 2 \\
1 ; 1
\end{array}\right)}{T^{2}}+\frac{6 \mathcal{L}_{2}\left(\begin{array}{l}
1 ; 1 ; 2 \\
1 ; 1 ; 1
\end{array}\right)}{T^{2}}+\frac{\mathcal{L}_{2}\left(\begin{array}{l}
1 ; 1 ; 1 ; 1 \\
1 ; 1 ; 1 ; 1
\end{array}\right)}{T^{2}}+\frac{4 \mathcal{L}_{3 / 2}\left(\begin{array}{l}
1 ; 3 \\
1 ; 1
\end{array}\right)}{T^{5 / 2}}+\frac{\mathcal{L}_{1}\left(\begin{array}{l}
4 \\
1
\end{array}\right)}{T^{3}}
$$

giving the expression for $C$. The orders of the various terms follow immediately. Finally, for each term the order of the expectation is $O\left(T^{-\mathcal{Q} / 2}\right)$ where $\mathcal{Q}$ is the number of score factors of the factor $\mathcal{L}_{\mathcal{P}}(R, P)$, giving $\mathbb{E}\left(\mathcal{L}_{1}\left(\begin{array}{l}2 \\ 1\end{array}\right) / l_{2}\right)=O(1), \mathbb{E}\left(\mathcal{L}_{1}\left(\begin{array}{l}1 ; 1 \\ 1 ; 1\end{array}\right) / l_{2}\right)=T^{-1}$, and so on.

Proof of Lemma 5 Consider the term $A_{1}$. Expanding $\widehat{\mathcal{L}}_{1}\left(\begin{array}{l}2 \\ 1\end{array}\right) / \widehat{l_{2}}$ around $\alpha$ and rearranging gives

$$
\frac{\mathcal{L}_{1}\left(\begin{array}{l}
2 \\
1
\end{array}\right)}{l_{2}}=\frac{\widehat{\mathcal{L}}_{1}\left(\begin{array}{l}
2 \\
1
\end{array}\right)}{\widehat{l}_{2}}-D_{1} V_{1}-\frac{1}{2} D_{2} V_{2}-\frac{1}{6} D_{3} V_{3}+O_{p}\left(T^{-2}\right)
$$

where $D_{j}=\partial_{\alpha}^{j}\left(\mathcal{L}_{1}\left(\begin{array}{l}2 \\ 1\end{array}\right) / l_{2}\right)$ and, by Lemma 2 ,

$$
\begin{aligned}
& V_{1}=a_{1 / 2}+a_{2 / 2}+a_{3 / 2}=-\frac{l_{1}}{l_{2}}-\frac{l_{1}^{2} l_{3}}{2 l_{2}^{3}}-\frac{l_{1}^{3} l_{3}^{2}}{2 l_{2}^{5}}+\frac{l_{1}^{3} l_{4}}{6 l_{2}^{4}}, \\
& V_{2}=a_{1 / 2}^{2}+2 a_{1 / 2} a_{2 / 2}=\frac{l_{1}^{2}}{l_{2}^{2}}+\frac{l_{1}^{3} l_{3}}{l_{2}^{4}}, \quad V_{3}=a_{1 / 2}^{3}=-\frac{l_{1}^{3}}{l_{2}^{3}} .
\end{aligned}
$$

Here, all terms with a factor $l_{1}^{3}$ are $[Z] / T^{3 / 2}+O_{p}\left(T^{-2}\right)$, so

$$
\begin{aligned}
& V_{1}=-\frac{l_{1}}{l_{2}}-\frac{l_{1}^{2} l_{3}}{2 l_{2}^{3}}+\frac{[Z]}{T^{3 / 2}}+O_{p}\left(T^{-2}\right), \\
& V_{2}=\frac{l_{1}^{2}}{l_{2}^{2}}+\frac{[Z]}{T^{3 / 2}}+O_{p}\left(T^{-2}\right), \quad V_{3}=\frac{[Z]}{T^{3 / 2}}+O_{p}\left(T^{-2}\right),
\end{aligned}
$$

and

$$
\begin{aligned}
A_{1} & =\frac{\widehat{\mathcal{L}}_{1}\left(\begin{array}{l}
2 \\
1
\end{array}\right)}{2 T \widehat{l}_{2}}-\frac{D_{1} V_{1}}{2 T}-\frac{D_{2} V_{2}}{4 T}-\frac{D_{3} V_{3}}{12 T}+O_{p}\left(T^{-3}\right) \\
& =\frac{\widehat{\mathcal{L}}_{1}\left(\begin{array}{l}
2 \\
1
\end{array}\right)}{2 T \widehat{l}_{2}}+\underbrace{\frac{D_{1} l_{1}}{2 T l_{2}}}_{A_{1.1}}+\underbrace{\frac{D_{1} l_{1}^{2} l_{3}}{4 T l_{2}^{3}}-\frac{D_{2} l_{1}^{2}}{4 T l_{2}^{2}}}_{A_{1.2}}+\frac{[Z]}{T^{5 / 2}}+O_{p}\left(T^{-3}\right) .
\end{aligned}
$$

Differentiating $\mathcal{L}_{1}\left(\begin{array}{l}2 \\ 1\end{array}\right)$ gives

$$
\partial_{\alpha} \mathcal{L}_{1}\left(\begin{array}{l}
2 \\
1
\end{array}\right)=2 \mathcal{L}_{1}\left(\begin{array}{l}
1,1 \\
1,2
\end{array}\right), \quad \partial_{\alpha}^{2} \mathcal{L}_{1}\left(\begin{array}{l}
2 \\
1
\end{array}\right)=2 \mathcal{L}_{1}\left(\begin{array}{l}
2 \\
2
\end{array}\right)+2 \mathcal{L}_{1}\left(\begin{array}{l}
1,1 \\
1,3
\end{array}\right),
$$

and, hence,

$$
\begin{aligned}
& D_{1}=\frac{2 \mathcal{L}_{1}\left(\begin{array}{l}
1,1 \\
1,2
\end{array}\right)}{l_{2}}-\frac{l_{3} \mathcal{L}_{1}\left(\begin{array}{l}
2 \\
1
\end{array}\right)}{l_{2}^{2}} \\
& D_{2}=\frac{2 \mathcal{L}_{1}\left(\begin{array}{l}
2 \\
2
\end{array}\right)}{l_{2}}+\frac{2 \mathcal{L}_{1}\left(\begin{array}{l}
1,1 \\
1,3
\end{array}\right)}{l_{2}}-\frac{l_{4} \mathcal{L}_{1}\left(\begin{array}{l}
2 \\
1
\end{array}\right)}{l_{2}^{2}}-\frac{4 l_{3} \mathcal{L}_{1}\left(\begin{array}{l}
1,1 \\
1,2
\end{array}\right)}{l_{2}^{2}}+\frac{2 l_{3}^{2} \mathcal{L}_{1}\left(\begin{array}{l}
2 \\
1
\end{array}\right)}{l_{2}^{3}}
\end{aligned}
$$


Therefore,

$$
\begin{aligned}
& A_{1.1}=\underbrace{\frac{l_{1} \mathcal{L}_{1}\left(\begin{array}{l}
1,1 \\
1,2
\end{array}\right)}{T l_{2}^{2}}-\frac{l_{3} l_{1} \mathcal{L}_{1}\left(\begin{array}{l}
2 \\
1
\end{array}\right)}{2 T l_{2}^{3}}}_{A_{1.1 .1}}, \\
& A_{1.2}=-\frac{l_{1}^{2} \mathcal{L}_{1}\left(\begin{array}{l}
2 \\
2
\end{array}\right)}{2 T l_{2}^{3}}-\frac{l_{1}^{2} \mathcal{L}_{1}\left(\begin{array}{l}
1,1 \\
1,3
\end{array}\right)}{2 T l_{2}^{3}}+\frac{l_{4} l_{1}^{2} \mathcal{L}_{1}\left(\begin{array}{l}
2 \\
1
\end{array}\right)}{4 T l_{2}^{4}}+\frac{3 l_{3} l_{1}^{2} \mathcal{L}_{1}\left(\begin{array}{l}
1,1 \\
1,2
\end{array}\right)}{2 T l_{2}^{4}}-\frac{3 l_{3}^{2} l_{1}^{2} \mathcal{L}_{1}\left(\begin{array}{l}
2 \\
1
\end{array}\right)}{4 T l_{2}^{5}} \text {. }
\end{aligned}
$$

Consider the term $A_{1.1 .1}$. The product rule gives

$$
A_{1.1 .1}=\frac{\mathcal{L}_{3 / 2}\left(\begin{array}{l}
1 ; 1,1 \\
1 ; 1,2
\end{array}\right)}{T^{3 / 2} l_{2}^{2}}+\frac{\mathcal{L}_{1}\left(\begin{array}{l}
2,1 \\
1,2
\end{array}\right)}{T^{2} l_{2}^{2}},
$$

where we need to further approximate the first term. Since $l_{2}^{2}=\left(\mathbb{E} l_{2}\right)^{2}+O_{p}\left(T^{-1 / 2}\right), 1 / l_{2}^{2}$ can be expanded as

$$
\frac{1}{l_{2}^{2}}=\frac{1}{\left(\mathbb{E} l_{2}\right)^{2}}-\frac{2\left(l_{2}-\mathbb{E} l_{2}\right)}{\left(\mathbb{E} l_{2}\right)^{3}}+\frac{3\left(l_{2}-\mathbb{E} l_{2}\right)^{2}}{\left(\mathbb{E} l_{2}\right)^{4}}+O_{p}\left(T^{-3 / 2}\right) .
$$

Therefore, since $\mathbb{E} \mathcal{L}_{3 / 2}\left(\begin{array}{l}1 ; 1,1 \\ 1 ; 1,2\end{array}\right)=0$,

$$
\frac{\mathcal{L}_{3 / 2}\left(\begin{array}{l}
1 ; 1,1 \\
1 ; 1,2
\end{array}\right)}{l_{2}^{2}}=-\frac{8 l_{2} \mathcal{L}_{3 / 2}\left(\begin{array}{l}
1 ; 1,1 \\
1 ; 1,2
\end{array}\right)}{\left(\mathbb{E} l_{2}\right)^{3}}+\frac{3 l_{2}^{2} \mathcal{L}_{3 / 2}\left(\begin{array}{l}
1 ; 1,1 \\
1 ; 1,2
\end{array}\right)}{\left(\mathbb{E} l_{2}\right)^{4}}+[Z]+O_{p}\left(T^{-3 / 2}\right),
$$

where, by the product rule,

$$
\begin{aligned}
l_{2} \mathcal{L}_{3 / 2}\left(\begin{array}{l}
1 ; 1,1 \\
1 ; 1,2
\end{array}\right) & =\frac{\mathcal{L}_{2}\left(\begin{array}{l}
1,1 ; 1,1,1 \\
1,2 ; 1,2
\end{array}\right)}{T^{1 / 2}}+\frac{\mathcal{L}_{3 / 2}\left(\begin{array}{l}
1 ; 1,2 \\
1 ; 1,2
\end{array}\right)}{T}+\mathcal{L}_{5 / 2}\left(\begin{array}{l}
1 ; 1 ; 1,1,1 \\
1 ; 2 ; 1,2
\end{array}\right) \\
& =\frac{\mathcal{L}_{2}\left(\begin{array}{l}
1,1 ; 1,1,1 \\
1,2 ; 1,2
\end{array}\right)}{T^{1 / 2}}+[Z], \\
l_{2}^{2} \mathcal{L}_{3 / 2}\left(\begin{array}{l}
1 ; 1,1 \\
1 ; 1,2
\end{array}\right) & =\mathcal{L}_{7 / 2}\left(\begin{array}{c}
1 ; 1 ; 1 ; 1,1 \\
1 ; 2 ; 2 ; 1,2
\end{array}\right)+\frac{2 \mathcal{L}_{3}\left(\begin{array}{l}
1 ; 1,1 ; 1 ; 1,1 \\
2 ; 1,2 ; 1,2
\end{array}\right)}{T^{1 / 2}}+\frac{2 \mathcal{L}_{5 / 2}\left(\begin{array}{l}
1 ; 1 ; 1,2 \\
1 ; 2 ; 1,2
\end{array}\right)}{T}+\frac{\mathcal{L}_{5 / 2}\left(\begin{array}{l}
1 ; 2 ; 1,1 \\
1 ; 2 ; 1,2
\end{array}\right)}{T}+O_{p}\left(T^{-3 / 2}\right) \\
& =\frac{2 \mathcal{L}_{3}\left(\begin{array}{l}
1 ; 1,1 ; 1 ; 1,1 \\
2 ; 1,2 ; 1,2
\end{array}\right)}{T^{1 / 2}}+[Z]+O_{p}\left(T^{-3 / 2}\right) .
\end{aligned}
$$

Hence, by Lemma 7,

$$
\begin{aligned}
\frac{\mathcal{L}_{3 / 2}\left(\begin{array}{l}
1 ; 1,1 \\
i 1,2
\end{array}\right)}{T^{3 / 2} l_{2}^{2}} & =-\frac{8 \mathcal{L}_{2}\left(\begin{array}{l}
1,1 ; 1 ; 1,1 \\
1,2 ; 1,2
\end{array}\right)}{T^{2}\left(\mathbb{E} l_{2}\right)^{3}}+\frac{6 \mathcal{L}_{3}\left(\begin{array}{l}
1 ; 1,1 ; 1,1,1 \\
2 ; 1,2 ; 1,2
\end{array}\right)}{T^{2}\left(\mathbb{E} l_{2}\right)^{4}}+\frac{[Z]}{T^{3 / 2}}+O_{p}\left(T^{-3}\right) \\
& =-\frac{2 \mathcal{L}_{2}\left(\begin{array}{l}
1,1 ; 1,1 \\
1,2 ; 1,2
\end{array}\right)}{T^{2}\left(\mathbb{E} l_{2}\right)^{3}}+\frac{[Z]}{T^{3 / 2}}+O_{p}\left(T^{-3}\right) \\
& =-\frac{2 \mathcal{L}_{1}\left(\begin{array}{l}
1,1 \\
1,2
\end{array}\right)}{T^{2} l_{2}^{3}}+\frac{[Z]}{T^{3 / 2}}+O_{p}\left(T^{-3}\right)
\end{aligned}
$$


and we obtain

$$
A_{1.1 .1}=-\frac{2 \mathcal{L}_{1}\left(\begin{array}{l}
1,1 \\
1,2
\end{array}\right)^{2}}{T^{2} l_{2}^{3}}+\frac{\mathcal{L}_{1}\left(\begin{array}{l}
2,1 \\
1,2
\end{array}\right)}{T^{2} l_{2}^{2}}+\frac{[Z]}{T^{3 / 2}}+O_{p}\left(T^{-3}\right) .
$$

Now consider $A_{1.1 .2}$. By the product rule,

$$
A_{1.1 .2}=-\frac{l_{3} \mathcal{L}_{3 / 2}\left(\begin{array}{c}
1 ; 2 \\
1 ; 1
\end{array}\right)}{2 T^{3 / 2} l_{2}^{3}}-\frac{l_{3} \mathcal{L}_{1}\left(\begin{array}{l}
3 \\
1
\end{array}\right)}{2 T^{2} l_{2}^{3}}
$$

where the first term has to be further approximated. Combining

$$
l_{3} \mathcal{L}_{3 / 2}\left(\begin{array}{c}
1 ; 2 \\
1 ; 1
\end{array}\right)=\mathcal{L}_{5 / 2}\left(\begin{array}{c}
1 ; 2 ; 1 \\
1 ; 1 ; 3
\end{array}\right)+\frac{\mathcal{L}_{2}\left(\begin{array}{l}
2 ; 1,1 \\
1 ; 1,3
\end{array}\right)}{T^{1 / 2}}+\frac{\mathcal{L}_{3 / 2}\left(\begin{array}{l}
1 ; 2,1 \\
1 ; 1,3
\end{array}\right)}{T}
$$

with the expansion

$$
\frac{1}{l_{2}^{3}}=\frac{1}{\left(\mathbb{E} l_{2}\right)^{3}}-\frac{3\left(l_{2}-\mathbb{E} l_{2}\right)}{\left(\mathbb{E} l_{2}\right)^{4}}+\frac{6\left(l_{2}-\mathbb{E} l_{2}\right)^{2}}{\left(\mathbb{E} l_{2}\right)^{5}}+O_{p}\left(T^{-3 / 2}\right)
$$

and $\mathbb{E} \mathcal{L}_{5 / 2}\left(\begin{array}{l}1 ; 2 ; 1 \\ 1 ; 1 ; 3\end{array}\right)=0$ gives

$$
\begin{aligned}
\frac{\mathcal{L}_{5 / 2}\left(\begin{array}{l}
1 ; 2 ; 1 \\
1 ; 1 ; 3
\end{array}\right)}{T^{3 / 2} l_{2}^{3}} & =-\frac{15 l_{2} \mathcal{L}_{5 / 2}\left(\begin{array}{l}
1 ; 2 ; 1 \\
1 ; 1 ; 3
\end{array}\right)}{T^{3 / 2}\left(\mathbb{E} l_{2}\right)^{4}}+\frac{6 l_{2}^{2} \mathcal{L}_{5 / 2}\left(\begin{array}{l}
1 ; 2 ; 1 ; 1 \\
(; 1 ; 3
\end{array}\right)}{T^{3 / 2}\left(\mathbb{E} l_{2}\right)^{5}}+\frac{[Z]}{T^{3 / 2}}+O_{p}\left(T^{-3}\right) \\
& =-\frac{15 \mathcal{L}_{3}\left(\begin{array}{l}
2 ; 1 ; 1,1,1 \\
1 ; 3 ; 1,2
\end{array}\right)}{T^{2}\left(\mathbb{E} l_{2}\right)^{4}}+\frac{12 \mathcal{L}_{3}\left(\begin{array}{l}
2 ; 1 ; 1,1 \\
1 ; 3 ; 1,2
\end{array}\right)}{T^{2}\left(\mathbb{E} l_{2}\right)^{4}}+\frac{[Z]}{T^{3 / 2}}+O_{p}\left(T^{-3}\right) \\
& =-\frac{3 \mathcal{L}_{3}\left(\begin{array}{l}
2 ; 1 ; 1,1 \\
1 ; 3 ; 1,2
\end{array}\right)}{T^{2}\left(\mathbb{E} l_{2}\right)^{4}}+\frac{[Z]}{T^{3 / 2}}+O_{p}\left(T^{-3}\right) \\
& =-\frac{3 l_{3} \mathcal{L}_{1}\left(\begin{array}{l}
2 \\
1
\end{array}\right) \mathcal{L}_{1}\left(\begin{array}{l}
1,1 \\
1,2
\end{array}\right)}{T^{2} l_{2}^{4}}+\frac{[Z]}{T^{3 / 2}}+O_{p}\left(T^{-3}\right),
\end{aligned}
$$

where we used

$$
\begin{aligned}
l_{2} \mathcal{L}_{5 / 2}\left(\begin{array}{l}
1 ; 2 ; 1 \\
1 ; 1 ; 3
\end{array}\right)= & \mathcal{L}_{7 / 2}\left(\begin{array}{l}
1 ; 2 ; 1 ; 1 \\
1 ; 1 ; 3 ; 2
\end{array}\right)+\frac{\mathcal{L}_{3}\left(\begin{array}{l}
2 ; 1 ; 1 ; 1,1 \\
1 ; 3 ; 1,2
\end{array}\right)}{T^{1 / 2}}+\frac{\mathcal{L}_{2 / 2}\left(\begin{array}{l}
1 ; 2 ; 1,1 \\
1 ; 1 ; 2,3
\end{array}\right)}{T}+\frac{\mathcal{L}_{5 / 2}\left(\begin{array}{l}
1 ; 1 ; 2,1 \\
1 ; 3 ; 1,2
\end{array}\right)}{T} \\
= & \frac{\mathcal{L}_{3}\left(\begin{array}{l}
2 ; 1 ; 1,1 \\
1 ; 3 ; 1,2
\end{array}\right)}{T^{1 / 2}}+[Z], \\
l_{2}^{2} \mathcal{L}_{5 / 2}\left(\begin{array}{l}
1 ; 2 ; 1 \\
1 ; 1 ; 3
\end{array}\right)= & \mathcal{L}_{9 / 2}\left(\begin{array}{l}
1 ; 2 ; 1 ; 1 ; 1 ; 1 ; 3 ; 2 ; 2 \\
1 ; 1 ;
\end{array}\right)+\frac{2 \mathcal{L}_{4}\left(\begin{array}{l}
2 ; 1 ; 1 ; 1,1 ; 1 ; 1 ; \\
T^{1 / 2}
\end{array}\right)}{T^{1 / 2}}+\frac{\mathcal{L}_{7 / 2}\left(\begin{array}{l}
1 ; 2 ; 1 ; 2 \\
1 ; 1 ; 3 ; 2
\end{array}\right)}{T}+\frac{2 \mathcal{L}_{7 / 2}\left(\begin{array}{l}
1 ; 2 ; 1 ; 1,1 \\
1 ; 1 ; 2 ; 2,3
\end{array}\right)}{T} \\
& +\frac{2 \mathcal{L}_{7 / 2}\left(\begin{array}{l}
1 ; 1 ; 1 ; 2,1 \\
1 ; 3 ; 2 ; 1,2
\end{array}\right)}{T}+O_{p}\left(T^{-3 / 2}\right) \\
= & \frac{2 \mathcal{L}_{4}\left(\begin{array}{c}
2 ; 1 ; 1 ; 1,1 \\
1 ; 3 ; 2 ; 1,2
\end{array}\right)}{T^{1 / 2}}+[Z]+O_{p}\left(T^{-3 / 2}\right)
\end{aligned}
$$

and Lemma 7. Further,

$$
\frac{\mathcal{L}_{2}\left(\begin{array}{l}
2 ; 1,1 \\
1 ; 1,3
\end{array}\right)}{T^{2} l_{2}^{3}}=\frac{4 \mathcal{L}_{2}\left(\begin{array}{l}
2 ; 1,1 \\
1 ; 1,3
\end{array}\right)}{T^{2}\left(\mathbb{E} l_{2}\right)^{3}}-\frac{3 l_{2} \mathcal{L}_{2}\left(\begin{array}{l}
2 ; 1,1 \\
1 ; 1,3
\end{array}\right)}{T^{2}\left(\mathbb{E} l_{2}\right)^{4}}+O_{p}\left(T^{-3}\right)
$$




$$
\begin{aligned}
& =\frac{4 \mathcal{L}_{1}\left(\begin{array}{l}
2 \\
1
\end{array}\right) \mathcal{L}_{1}\left(\begin{array}{l}
1,1 \\
1,3
\end{array}\right)}{T^{2} l_{2}^{3}}-\frac{3 \mathcal{L}_{1}\left(\begin{array}{l}
2 \\
1
\end{array}\right) \mathcal{L}_{2}\left(\begin{array}{l}
1,1 \\
1,3
\end{array}\right)}{T^{2} l_{2}^{3}}+O_{p}\left(T^{-3}\right) \\
& =\frac{\mathcal{L}_{1}\left(\begin{array}{l}
2 \\
1
\end{array}\right) \mathcal{L}_{1}\left(\begin{array}{l}
1,1 \\
1,3
\end{array}\right)}{T^{2} l_{2}^{3}}+O_{p}\left(T^{-3}\right)
\end{aligned}
$$

and, since $\mathbb{E} \mathcal{L}_{3 / 2}\left(\begin{array}{c}1 ; 2,1 \\ 1 ; 1,3\end{array}\right)=0$,

$$
\frac{\mathcal{L}_{3 / 2}\left(\begin{array}{c}
1 ; 2,1 \\
1 ; 1,3
\end{array}\right)}{T^{5 / 2} l_{2}^{3}}=\frac{[Z]}{T^{5 / 2}}+O_{p}\left(T^{-3}\right)
$$

It follows that

$$
A_{1.1 .2}=-\frac{\mathcal{L}_{1}\left(\begin{array}{l}
2 \\
1
\end{array}\right) \mathcal{L}_{1}\left(\begin{array}{l}
1,1 \\
1,3
\end{array}\right)}{2 T^{2} l_{2}^{3}}-\frac{l_{3} \mathcal{L}_{1}\left(\begin{array}{l}
3 \\
1
\end{array}\right)}{2 T^{2} l_{2}^{3}}+\frac{3 l_{3} \mathcal{L}_{1}\left(\begin{array}{l}
2 \\
1
\end{array}\right) \mathcal{L}_{1}\left(\begin{array}{l}
1,1 \\
1,2
\end{array}\right)}{2 T^{2} l_{2}^{4}}+\frac{[Z]}{T^{3 / 2}}+O_{p}\left(T^{-3}\right)
$$

Consider $A_{1.2}$. Recalling $l_{1}^{2}=\mathcal{L}_{1}\left(\begin{array}{l}2 \\ 1\end{array}\right) / T+\mathcal{L}_{1}\left(\begin{array}{c}1 ; 1 \\ 1 ; 1\end{array}\right) / T$, we have

$$
\begin{aligned}
l_{1}^{2} \mathcal{L}_{1}\left(\begin{array}{l}
2 \\
2
\end{array}\right) & =\frac{\mathcal{L}_{1}\left(\begin{array}{l}
2 \\
1
\end{array}\right) \mathcal{L}_{1}\left(\begin{array}{l}
2 \\
2
\end{array}\right)}{T}+\frac{[Z]}{T} \\
& =\frac{\mathcal{L}_{2}\left(\begin{array}{l}
2 ; 2 \\
1 ; 2
\end{array}\right)}{T}+\frac{\mathcal{L}_{1}\left(\begin{array}{l}
2,2 \\
1,2
\end{array}\right)}{T^{2}}+\frac{[Z]}{T} \\
& =\frac{\mathcal{L}_{1}\left(\begin{array}{l}
2 \\
1
\end{array}\right) \mathcal{L}_{1}\left(\begin{array}{l}
2 \\
2
\end{array}\right)}{T}+\frac{[Z]}{T}+O_{p}\left(T^{-2}\right)
\end{aligned}
$$

and, similarly,

$$
\begin{aligned}
l_{1}^{2} \mathcal{L}_{1}\left(\begin{array}{l}
2 \\
1
\end{array}\right) & =\frac{\mathcal{L}_{1}\left(\begin{array}{l}
2 \\
1
\end{array}\right)^{2}}{T}+\frac{[Z]}{T}+O_{p}\left(T^{-2}\right), \\
l_{1}^{2} \mathcal{L}_{1}\left(\begin{array}{l}
1,1 \\
1,2
\end{array}\right) & =\frac{\mathcal{L}_{1}\left(\begin{array}{l}
2 \\
1
\end{array}\right) \mathcal{L}_{1}\left(\begin{array}{l}
1,1 \\
1,2
\end{array}\right)}{T}+\frac{[Z]}{T}+O_{p}\left(T^{-2}\right), \\
l_{1}^{2} \mathcal{L}_{1}\left(\begin{array}{l}
1,1 \\
1,3
\end{array}\right) & =\frac{\mathcal{L}_{1}\left(\begin{array}{l}
2 \\
1
\end{array}\right) \mathcal{L}_{1}\left(\begin{array}{l}
1,1 \\
1,3
\end{array}\right)}{T}+\frac{[Z]}{T}+O_{p}\left(T^{-2}\right) .
\end{aligned}
$$

Therefore

$$
A_{1.2}=-\frac{\mathcal{L}_{1}\left(\begin{array}{l}
2 \\
1
\end{array}\right) \mathcal{L}_{1}\left(\begin{array}{l}
2 \\
2
\end{array}\right)}{2 T^{2} l_{2}^{3}}-\frac{\mathcal{L}_{1}\left(\begin{array}{l}
2 \\
1
\end{array}\right) \mathcal{L}_{1}\left(\begin{array}{l}
1,1 \\
1,3
\end{array}\right)}{2 T^{2} l_{2}^{3}}+\frac{l_{4} \mathcal{L}_{1}\left(\begin{array}{l}
2 \\
1
\end{array}\right)^{2}}{4 T^{2} l_{2}^{4}}+\frac{3 l_{3} \mathcal{L}_{1}\left(\begin{array}{l}
2 \\
1
\end{array}\right) \mathcal{L}_{1}\left(\begin{array}{l}
1,1 \\
1,2
\end{array}\right)}{2 T^{2} l_{2}^{4}}-\frac{3 l_{3}^{2} \mathcal{L}_{1}\left(\begin{array}{l}
2 \\
1
\end{array}\right)^{2}}{4 T^{2} l_{2}^{5}}+\frac{[Z]}{T^{2}}+O_{p}\left(T^{-3}\right)
$$

and the expression for $A_{1}$ follows from (5.9)-(5.14). Now consider $A_{2}$. From

$$
\frac{1}{l_{2}}=\frac{1}{\mathbb{E} l_{2}}-\frac{l_{2}-\mathbb{E} l_{2}}{\left(\mathbb{E} l_{2}\right)^{2}}+\frac{\left(l_{2}-\mathbb{E} l_{2}\right)^{2}}{\left(\mathbb{E} l_{2}\right)^{3}}-\frac{\left(l_{2}-\mathbb{E} l_{2}\right)^{3}}{\left(\mathbb{E} l_{2}\right)^{4}}+O_{p}\left(T^{-2}\right)
$$

it follows that

$$
A_{2}=\underbrace{\frac{\mathcal{L}_{1}\left(\begin{array}{l}
1 ; 1 \\
1 ; 1
\end{array}\right)}{2 T \mathbb{E} l_{2}}}_{A_{2.1}}-\underbrace{\frac{\left(l_{2}-\mathbb{E} l_{2}\right) \mathcal{L}_{1}\left(\begin{array}{l}
1 ; 1 \\
1 ; 1
\end{array}\right)}{2 T\left(\mathbb{E} l_{2}\right)^{2}}}_{A_{2.2}}+\underbrace{\frac{\left(l_{2}-\mathbb{E} l_{2}\right)^{2} \mathcal{L}_{1}\left(\begin{array}{l}
1 ; 1 \\
1 ; 1
\end{array}\right)}{2 T\left(\mathbb{E} l_{2}\right)^{3}}}_{A_{2.3}}-\underbrace{\frac{\left(l_{2}-\mathbb{E} l_{2}\right)^{3} \mathcal{L}_{1}\left(\begin{array}{l}
1 ; 1 \\
1 ; 1
\end{array}\right)}{2 T\left(\mathbb{E} l_{2}\right)^{4}}}_{A_{2.4}}+O_{p}\left(T^{-3}\right),
$$


where, by Lemma $\mathbf{6}$,

$$
A_{2.1}=\frac{[Z]}{T}, \quad A_{2.2}=\frac{[Z]}{T}, \quad A_{2.3}=\frac{l_{2}^{2} \mathcal{L}_{1}\left(\begin{array}{c}
1 ; 1 \\
1 ; 1
\end{array}\right)}{2 T\left(\mathbb{E} l_{2}\right)^{3}}+\frac{[Z]}{T}, \quad A_{2.4}=\frac{l_{2}^{3} \mathcal{L}_{1}\left(\begin{array}{c}
1 ; 1 \\
1 ; 1
\end{array}\right)}{2 T\left(\mathbb{E} l_{2}\right)^{4}}-\frac{3 l_{2}^{2} \mathcal{L}_{1}\left(\begin{array}{l}
1 ; 1 \\
1 ; 1
\end{array}\right)}{2 T\left(\mathbb{E} l_{2}\right)^{3}}+\frac{[Z]}{T} .
$$

Recursive application of the product rule gives

$$
\begin{aligned}
l_{2}^{2} \mathcal{L}_{1}\left(\begin{array}{l}
1 ; 1 \\
1 ; 1
\end{array}\right)= & \mathcal{L}_{3}\left(\begin{array}{l}
1 ; 1 ; 1 ; 1 \\
1 ; 1 ; 2 ; 2
\end{array}\right)+\frac{4 \mathcal{L}_{5 / 2}\left(\begin{array}{l}
1 ; 1 ; 1,1 \\
1 ; 2 ; 1,2
\end{array}\right)}{T^{1 / 2}}+\frac{2 \mathcal{L}_{2}\left(\begin{array}{l}
1,1 ; 1 ; 1 \\
1,2 ; 1,2
\end{array}\right)}{T}+\frac{\mathcal{L}_{2}\left(\begin{array}{l}
1 ; 1 ; 2 \\
1 ; 1 ; 2
\end{array}\right)}{T}+\frac{2 \mathcal{L}_{3 / 2}\left(\begin{array}{l}
1 ; 1,2 \\
1 ; 1,2
\end{array}\right)}{T^{3 / 2}} \\
= & \frac{2 \mathcal{L}_{2}\left(\begin{array}{l}
1,1 ; 1,1 \\
1,2 ; 1,2
\end{array}\right)}{T}+[Z]=\frac{2 \mathcal{L}_{1}\left(\begin{array}{l}
1,1 \\
1,2
\end{array}\right)}{T}+[Z]+O_{p}\left(T^{-2}\right), \\
l_{2}^{3} \mathcal{L}_{1}\left(\begin{array}{l}
1 ; 1 \\
1 ; 1
\end{array}\right)= & \mathcal{L}_{4}\left(\begin{array}{l}
1 ; 1 ; 1 ; 1 ; 1 \\
1 ; 1 ; 2 ; 2 ; 2
\end{array}\right)+\frac{6 \mathcal{L}_{7 / 2}\left(\begin{array}{l}
1 ; 1 ; 1 ; 1,1 \\
1 ; 2 ; 2 ; 1,2
\end{array}\right)}{T^{1 / 2}}+\frac{3 \mathcal{L}_{3}\left(\begin{array}{l}
1 ; 1 ; 1 ; 2 \\
1 ; 1 ; 2 ; 2
\end{array}\right)}{T}+\frac{6 \mathcal{L}_{3}\left(\begin{array}{l}
1 ; 1,1 ; 1,1 \\
2 ; 1 ; 2 ; 1,2
\end{array}\right)}{T}+\frac{6 \mathcal{L}_{5 / 2}\left(\begin{array}{l}
1 ; 2 ; 1,1 \\
1 ; 2 ; 1,2
\end{array}\right)}{T^{3 / 2}} \\
& +\frac{6 \mathcal{L}_{5 / 2}\left(\begin{array}{l}
1 ; 1 ; 1,2 \\
1 ; 2 ; 1,2
\end{array}\right)}{T^{3 / 2}}+\frac{6 \mathcal{L}_{2}\left(\begin{array}{l}
1,1 ; 1 ; 1,2 \\
1,2 ; 1,2
\end{array}\right)}{T^{2}}+\frac{\mathcal{L}_{2}\left(\begin{array}{l}
1 ; 1 ; 3 \\
1 ; 1 ; 2
\end{array}\right)}{T^{2}}+\frac{2 \mathcal{L}_{3 / 2}\left(\begin{array}{l}
1 ; 1 ; 3 \\
1 ; 1,2
\end{array}\right)}{T^{5 / 2}} \\
= & \frac{6 \mathcal{L}_{3}\left(\begin{array}{l}
1 ; 1,1 ; 1,1 \\
2 ; 1,2 ; 1,2
\end{array}\right)}{T}+[Z]+O_{p}\left(T^{-2}\right)=\frac{6 \mathcal{L}_{1}\left(\begin{array}{l}
1 \\
2
\end{array}\right) \mathcal{L}_{1}\left(\begin{array}{l}
1,1 \\
1,2
\end{array}\right)}{T}+[Z]+O_{p}\left(T^{-2}\right) .
\end{aligned}
$$

Hence

$$
\begin{aligned}
& A_{2.3}=\frac{\mathcal{L}_{1}\left(\begin{array}{l}
1,1 \\
1,2
\end{array}\right)^{2}}{T^{2}\left(\mathbb{E} l_{2}\right)^{3}}+\frac{[Z]}{T}+O_{p}\left(T^{-3}\right)=\frac{\mathcal{L}_{1}\left(\begin{array}{l}
1,1 \\
1,2
\end{array}\right)^{2}}{T^{2} l_{2}^{3}}+\frac{[Z]}{T}+O_{p}\left(T^{-3}\right), \\
& A_{2.4}=\frac{3 \mathcal{L}_{1}\left(\begin{array}{l}
1 \\
2
\end{array}\right) \mathcal{L}_{1}\left(\begin{array}{l}
1,1 \\
1,2
\end{array}\right)^{2}}{T^{2}\left(\mathbb{E} l_{2}\right)^{4}}-\frac{3 \mathcal{L}_{1}\left(\begin{array}{l}
1,1 \\
1,2
\end{array}\right)^{2}}{T^{2}\left(\mathbb{E} l_{2}\right)^{3}}+\frac{[Z]}{T}+O_{p}\left(T^{-3}\right)=\frac{[Z]}{T}+O_{p}\left(T^{-3}\right),
\end{aligned}
$$

and the expression for $A_{2}$ follows. For $B_{1}$, from (5.12) and Lemma 6 , we have

$$
B_{1}=-\frac{15 l_{2} \mathcal{L}_{5 / 2}\left(\begin{array}{l}
1 ; 2 ; 1 \\
1 ; 1 ; 3
\end{array}\right)}{2 T^{3 / 2}\left(\mathbb{E} l_{2}\right)^{4}}+\frac{3 l_{2}^{2} \mathcal{L}_{5 / 2}\left(\begin{array}{l}
1 ; 2 ; 1 ; \\
1 ; 1 ; 3
\end{array}\right)}{T^{3 / 2}\left(\mathbb{E} l_{2}\right)^{5}}+\frac{[Z]}{T^{3 / 2}}+O_{p}\left(T^{-3}\right)
$$

By the product rule,

$$
\begin{aligned}
& l_{2} \mathcal{L}_{5 / 2}\left(\begin{array}{l}
1 ; 2 ; 1 \\
1 ; 1 ; 3
\end{array}\right)=\mathcal{L}_{7 / 2}\left(\begin{array}{l}
1 ; 2 ; 1 ; 1 \\
1 ; 1 ; 3 ; 2
\end{array}\right)+\frac{\mathcal{L}_{3}\left(\begin{array}{l}
2 ; 1 ; 1,1 \\
1 ; 3 ; 1,2
\end{array}\right)}{T^{1 / 2}}+\frac{\mathcal{L}_{5 / 2}\left(\begin{array}{l}
1 ; 2 ; 1 ; 1 \\
1 ; 1 ; 2,3
\end{array}\right)}{T}+\frac{\mathcal{L}_{5 / 2}\left(\begin{array}{l}
1 ; 1 ; 2,1 \\
1 ; 3 ; 1,2
\end{array}\right)}{T} \\
& =\frac{\mathcal{L}_{3}\left(\begin{array}{c}
2 ; 1 ; 1,1 \\
1 ; 3 ; 1,2
\end{array}\right)}{T^{1 / 2}}+[Z] \\
& l_{2}^{2} \mathcal{L}_{5 / 2}\left(\begin{array}{c}
1 ; 2 ; 1 \\
1 ; 1 ; 3
\end{array}\right)=\mathcal{L}_{9 / 2}\left(\begin{array}{l}
1 ; 2 ; 1 ; 1 ; 1 \\
1 ; 1 ; 3 ; 2 ; 2
\end{array}\right)+\frac{2 \mathcal{L}_{4}\left(\begin{array}{l}
2 ; 1 ; 1 ; 1,1 \\
1 ; 3 ; ; ; 1,2
\end{array}\right)}{T^{1 / 2}}+\frac{\mathcal{L}_{7 / 2}\left(\begin{array}{l}
1 ; 2 ; 1 ; 2 \\
1 ; 1 ; 3 ; 2
\end{array}\right)}{T}+\frac{2 \mathcal{L}_{7 / 2}\left(\begin{array}{l}
1 ; 2 ; 1 ; 1,1 \\
1 ; 1 ; 2 ; 2,3
\end{array}\right)}{T} \\
& +\frac{2 \mathcal{L}_{7 / 2}\left(\begin{array}{c}
1 ; 1 ; 1 ; 2,1 \\
1 ; 3 ; 2 ; 1,2
\end{array}\right)}{T}+O_{p}\left(T^{-3 / 2}\right) \\
& =\frac{2 \mathcal{L}_{4}\left(\begin{array}{l}
2 ; 1 ; 1 ; 1 ; 1 \\
1 ; 3 ; 2 ; 1,2
\end{array}\right)}{T^{1 / 2}}+[Z]+O_{p}\left(T^{-3 / 2}\right) .
\end{aligned}
$$


Hence

$$
\begin{aligned}
B_{1} & =-\frac{15 \mathcal{L}_{3}\left(\begin{array}{l}
2 ; 1 ; 1 ; 1,1 \\
1 ; 3 ; 1,2
\end{array}\right)}{2 T^{2}\left(\mathbb{E} l_{2}\right)^{4}}+\frac{6 \mathcal{L}_{4}\left(\begin{array}{l}
2 ; 1 ; 1 ; 1 ; 1,1 \\
1 ; 3 ; 2 ; 1,2
\end{array}\right)}{T^{2}\left(\mathbb{E} l_{2}\right)^{5}}+\frac{[Z]}{T^{3 / 2}}+O_{p}\left(T^{-3}\right) \\
& =-\frac{15 \mathcal{L}_{3}\left(\begin{array}{l}
2 ; 1 ; 1,1 \\
1 ; 3 ; 1,2
\end{array}\right)}{2 T^{2}\left(\mathbb{E} l_{2}\right)^{4}}+\frac{6 \mathcal{L}_{3}\left(\begin{array}{l}
2 ; 1 ; 1,1 \\
1 ; 3 ; 1,2
\end{array}\right)}{T^{2}\left(\mathbb{E} l_{2}\right)^{4}}+\frac{[Z]}{T^{3 / 2}}+O_{p}\left(T^{-3}\right) \\
& =-\frac{3 \mathcal{L}_{1}\left(\begin{array}{l}
2 \\
1
\end{array}\right) \mathcal{L}_{1}\left(\begin{array}{l}
1 \\
3
\end{array}\right) \mathcal{L}_{1}\left(\begin{array}{l}
1,1 \\
1,2
\end{array}\right)}{2 T^{2} l_{2}^{4}}+\frac{[Z]}{T^{3 / 2}}+O_{p}\left(T^{-3}\right) .
\end{aligned}
$$

Finally, $B_{2}$ and $C_{1}$ follow from

$$
\begin{aligned}
\mathcal{L}_{2}\left(\begin{array}{l}
2 ; 1,1 \\
1 ; 1,3
\end{array}\right) & =\mathcal{L}_{1}\left(\begin{array}{l}
2 \\
1
\end{array}\right) \mathcal{L}_{1}\left(\begin{array}{l}
1,1 \\
1,3
\end{array}\right)+O_{p}\left(T^{-1}\right), \\
\mathcal{L}_{2}\left(\begin{array}{l}
2 ; 2 \\
1 ; 1
\end{array}\right) & =\mathcal{L}_{1}\left(\begin{array}{l}
2 \\
1
\end{array}\right)^{2}+O_{p}\left(T^{-1}\right) .
\end{aligned}
$$

\section{References}

Andersen, E. B. (1970). Asymptotic properties of conditional maximum-likelihood estimators. Journal of the Royal Statistical Society. Series B (Methodological) 32(2), 283-301.

Anderson, T. W. and C. Hsiao (1982). Formulation and estimation of dynamic models using panel data. Journal of econometrics 18(1), 47-82.

Arellano, M. and S. Bond (1991). Some tests of specification for panel data: Monte Carlo evidence and an application to employment equations. The Review of Economic Studies 58(2), 277-297.

Arellano, M. and S. Bonhomme (2009). Robust priors in nonlinear panel data models. Econometrica $77(2), 489-536$.

Arellano, M. and J. Hahn (2016). A likelihood-based approximate solution to the incidental parameter problem in dynamic nonlinear models with multiple effects. Global Economic Review 45(3), $251-274$.

Bester, C. A. and C. Hansen (2009). A penalty function approach to bias reduction in nonlinear panel models with fixed effects. Journal of Business $8 \mathcal{G}$ Economic Statistics 27(2), 131-148.

Blundell, R., R. Griffith, and F. Windmeijer (2002). Individual effects and dynamics in count data models. Journal of Econometrics 108(1), 113-131.

Bonhomme, S. (2012). Functional differencing. Econometrica 80(4), 1337-1385.

Chamberlain, G. (1980). Analysis of covariance with qualitative data. The Review of Economic Studies 47(1), 225-238.

Chamberlain, G. (2010). Binary response models for panel data: Identification and information. Econometrica 78(1), 159-168. 
Chamberlain, G. and M. J. Moreira (2009). Decision theory applied to a linear panel data model. Econometrica $77(1), 107-133$.

Cox, D. (1958). Two further applications of a model for binary regression. Biometrika 45(3/4), $562-565$.

Cox, D. R. and E. J. Snell (1968). A general definition of residuals. Journal of the Royal Statistical Society. Series B (Methodological) 30(2), 248-275.

Dhaene, G. and K. Jochmans (2015). Split-panel jackknife estimation of fixed-effect models. The Review of Economic Studies 82(3), 991-1030.

Dhaene, G. and K. Jochmans (2016). Likelihood inference in an autoregression with fixed effects. Econometric Theory 32(5), 1178-1215.

Dhaene, G. and K. Jochmans (2017). Profile-score adjustments for incidental-parameter problems. Working paper.

Fernández-Val, I. (2009). Fixed effects estimation of structural parameters and marginal effects in panel probit models. Journal of Econometrics 150(1), 71-85.

Greene, W. (2004). The behaviour of the maximum likelihood estimator of limited dependent variable models in the presence of fixed effects. The Econometrics Journal 7(1), 98-119.

Hahn, J. and G. Kuersteiner (2002). Asymptotically unbiased inference for a dynamic panel model with fixed effects when both n and T are large. Econometrica 70(4), 1639-1657.

Hahn, J. and G. Kuersteiner (2011). Bias reduction for dynamic nonlinear panel models with fixed effects. Econometric Theory 27(06), 1152-1191.

Hahn, J. and W. Newey (2004). Jackknife and analytical bias reduction for nonlinear panel models. Econometrica 72(4), 1295-1319.

Heckman, J. (1981). The incidental parameters problem and the problem of initial conditions in estimating a discrete time-discrete data stochastic process. In C. Manski and D. McFadden (Eds.), Structural Analysis of Discrete Data. MIT Press.

Honoré, B. E. (1992). Trimmed LAD and least squares estimation of truncated and censored regression models with fixed effects. Econometrica 60, 533-565.

Kalbfleisch, J. D. and D. A. Sprott (1970). Application of likelihood methods to models involving large numbers of parameters. Journal of the Royal Statistical Society. Series B (Methodological) 1, $175-208$.

Kim, M. S. and Y. Sun (2016). Bootstrap and k-step bootstrap bias corrections for the fixed effects estimator in nonlinear panel data models. Econometric Theory 32(6), 1523-1568.

Lancaster, T. (2000). The incidental parameter problem since 1948. Journal of Econometrics 95(2), 391-413.

Lancaster, T. (2002). Orthogonal parameters and panel data. The Review of Economic Studies $69(3), 647-666$. 
Manski, C. F. (1987). Semiparametric analysis of random effects linear models from binary panel data. Econometrica 55(2), 357-362.

Moreira, M. (2009). A maximum likelihood method for the incidental parameter problem. The Annals of Statistics 37(6A), 3660-3696.

Newey, W. K. and D. McFadden (1994). Large sample estimation and hypothesis testing. In J. J. Heckman and L. E. E. (Eds.), Handbook of Econometrics (Vol. 4), Chapter 36, pp. 2111-2245. Elsevier.

Neyman, J. and E. L. Scott (1948). Consistent estimates based on partially consistent observations. Econometrica 16(1), 1-32.

Nickell, S. (1981). Biases in dynamic models with fixed effects. Econometrica 49(6), 1417-1426.

Pace, L. and A. Salvan (1997). Principles of statistical inference from a neo-Fisherian perspective, Volume 4. World Scientific.

Pace, L. and A. Salvan (2006). Adjustments of the profile likelihood from a new perspective. Journal of Statistical Planning and Inference 136(10), 3554-3564.

Woutersen, T. (2002). Robustness against incidental parameters and mixing distributions. Unpublished manuscript. 